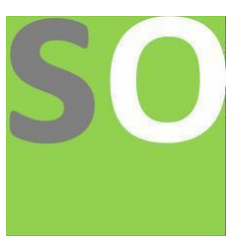

Article title: This article has been taken down

Authors: ROLAND ABI[1], A.F Fagbamigbe[2], A.M Akinwande[3]

Affiliations: Department of Epidemiology and Medical Statistics, University of Ibadan, Ibadan, Nigeria[1], Department of Radiation Oncology, University College Hospital, Ibadan, Nigeria[2]

Orcid ids: 0000-0001-8550-802X[1]

Contact e-mail: edame12@gmail.com

License information: This work has been published open access under Creative Commons Attribution License http://creativecommons.org/licenses/by/4.0/, which permits unrestricted use, distribution, and reproduction in any medium, provided the original work is properly cited. Conditions, terms of use and publishing policy can be found at https://www.scienceopen.com/.

Preprint statement: This article is a preprint and has not been peer-reviewed, under consideration and submitted to ScienceOpen Preprints for open peer review.

DOI: 10.14293/S2199-1006.1.SOR-.PPCCW4P.v1

Preprint first posted online: 01 December 2020

Keywords: Prostate cancer 


\title{
COMPARISON OF BAYESIAN AND FREQUENTIST SURVIVAL ANALYSIS METHODS IN MODELLING OF PROSTATE CANCER SURVIVORSHIP IN OYO STATE, NIGERIA
}

Abi Roland, Fagbamigbe Adeniyi, Akinwande Mathew

\begin{abstract}
Background: Survival analysis is a statistical method for modelling the probability that a subset of a given population will survive past a certain time. In the context of cancer, this probability would represent recurrence of tumour, or remission (i.e. being disease-free). This study seeks to compare the traditional frequentist approach and the Bayesian approach to survival analysis in estimating, and the predictors of prostate cancer $(\mathrm{CaP})$ survivorship. Prostate cancer starts when healthy cells in the prostate gland change and grow out of place, forming a mass called a tumour.
\end{abstract}

Method: A retrospective analytical study design was employed, through extraction of case files patients diagnosed and treated for CaP from January 2010 to December 2017 at UCH, Ibadan. The extracted data was further divided into two cohorts (2010 - 2014) and (2015 - 2017). A proforma was used for extraction which includes the following sections; socio-demographics, clinical/pathological characteristics, date of diagnosis, date last seen, and treatment received. Descriptive statistics was used to describe these characteristics. The survival probability was determined by the KM survival method. Cox proportional hazard (CPH), Weibull AFT and Bayesian Weibull AFT (using normal prior distribution) models was used to determine predictors of survivorship.

Results: The average age of the patients was 72 years, with peak incidence of $\mathrm{CaP}$ among those aged $70-79$ years. Most patients $87.3 \%$ were diagnosed at stage IV, with many having metastasis to the spine. Among the patients, 33.6\% received chemotherapy and surgery. Patients from North central had the highest median survival (MS) time in the (2015 - 2017) cohort. The overall MS time for the (2010 - 2014) cohort was 2.9 months, and 3.3 months for the (2015 - 2017) cohort while the overall MS time for the study was 3.2 months. Patients treated with chemotherapy and 
surgery in both cohorts experienced delayed remission. The Weibull AFT model found that patients with a moderately differentiated Gleason experienced a 50\% increase in time ( $\mathrm{TR}=0.5$; 95\%CI: $0.3-0.9)$ to remission. Patients, with a poorly differentiated Gleason, experienced a 70\% decrease in time (aTR $=1.7 ; 95 \% \mathrm{CI}: 1.0-2.7)$ to remission. The Bayesian AFT model also found delay in time to remission for patients with moderately differentiated Gleason (TR $=0.6$; 95\% CrI: $0.3-0.9$ ), and those treated with Chemotherapy and Surgery (aTR $=3.3 ; 95 \%$ CrI: $2.6-4.4$ ). The Bayesian model showed that age, south-south, north-central, no family history of $\mathrm{CaP}$, moderately and poor differentiated Gleason and treatment with Chemotherapy, Radiotherapy and Surgery, Chemotherapy and Surgery to significantly delay time to remission.

Conclusion: This study found that in considering predictors of survivorship a host of factors should be considered, particularly age, location, marital status, occupation, stage, method of diagnosis, Gleason group, site and treatment received. In terms of approaches to survival analysis, greater emphasis should be given to the Bayesian approach, as observed in this study, the Bayesian approach extracted more significant predictors of survivorship than the CPH and Weibull AFT models and besides, it is more suitable for studies with fewer observations.

Keywords: survival analysis, parametric, frequentist, Bayesian, prostate cancer. 


\section{Introduction}

Survival analysis is a method for modeling time duration to the occurrence or recurrence of events. It is also called reliability theory or reliability analysis in the field of engineering. In general, survival analysis is a statistical modeling approach that involves time to event data. An important aspect of survival analysis data is censoring and non-normality. Non-normality of the data violates the normality assumption of most commonly used statistical method such as multiple linear regressions (Oakes, 1972). The objective of using survival analysis is to answer the question of, what is the probability that subset of a given population will survive past a certain time? In the context of cancer, this probability would represent death, relapse, recurrence of the tumour, or remission (i.e. being disease-free) of a cancer patient.

\section{Background/rationale}

Frequentist methodology of survival analysis

Much of the development of frequentist methodology is attributed to the work carried out by R.A. Fisher in the early 20th century. The frequentist approach to statistics evaluates procedures based on imagining repeated sampling from a particular model (the likelihood), which defines the probability distribution of the observed data conditional on unknown parameters. Properties of the procedure are evaluated in this repeated sampling framework for fixed values of unknown parameters; good procedures perform well over a broad range of parameter values (Gandenberger, 2014a, 2014b, 2014c). Survival analysis in the frequentist framework is based on non-parametric methods (Nelson Aalen, Kaplan Meier), Semi-parametric (Cox Proportional Hazard) and the parametric (Weibull, Exponential, Log-logistic etc) models.

\section{Bayesian methodology of survival analysis}

The introduction of Bayesian methodology can be attributed to a posthumous paper titled "An essay towards solving the Problem in the Doctrine of Chances" by the Reverend Thomas Bayes in 1763 (Bayes, 1763). Despite its early inception, frequentist methods remain the dominant methodology in modern statistics. In part, this was due to the lack of modern sampling techniques that require substantial computing power (Ibrahim, Chen, \& Sinha, 2005). Indeed, for all but the simplest Bayesian models, computation methods were complex and was often impossible to find 
any analytical solutions. Whilst advances in theory and computing have made Bayesian methods more accessible, their uses in practice still remain somewhat limited.

In estimating the parameter $\boldsymbol{\theta}$ associated with data $\boldsymbol{x}$, under the Bayesian approach, $P(x \mid \theta)$ is evaluated where $P($.$) represents some probability density (Jackson, 2015). Bayesian methodology$ considers the data, once observed, to be a fixed quantity and allows the parameters to be the random variables; thus, instead evaluating $P(\boldsymbol{x} \mid \boldsymbol{\theta})$.

Evaluation of $P(\boldsymbol{x} \mid \boldsymbol{\theta})$ is determined via use of Bayes' theorem for conditional probability

$$
P(\boldsymbol{\theta} \mid \boldsymbol{x})=\frac{P(\boldsymbol{x} \mid \boldsymbol{\theta}) P(\boldsymbol{\theta})}{P(\boldsymbol{x})}
$$

Under conventional notation, define

$$
\begin{gathered}
P(\boldsymbol{\theta} \mid \boldsymbol{x})=\text { Posterior probability; } P(\boldsymbol{x} \mid \boldsymbol{\theta})=\text { Likelihood; } P(\boldsymbol{\theta})=\text { Prior probability } ; \\
P(\boldsymbol{x})=\text { margnial probability }
\end{gathered}
$$

All inferences are made on summaries of the posterior probability density, the posterior is a product of the information obtained from the data and the information taken from prior knowledge. Note that the marginal density $P(\boldsymbol{x})$ is simply the probability of observing the data which is not dependent upon the parameter $\boldsymbol{\theta}$ (Berger, 1985; Box \& Tiao, 1992).

Prostate cancer starts when healthy cells in the prostate gland change and grows out of place, forming a mass called a tumour. (ASCO, 2015) The Prostate is a walnut-sized organ located just between the bladder and the male reproductive organ, and it slowly grows larger to an average weight of 40 grams in ageing men. A prostate gland surrounds the urethra that empties urine from the bladder and also secretes prostate fluid that protects sperm. These physiological functions may be compromised during various prostate diseases including prostatitis, benign prostatic hyperplasia or hypertrophy, and cancer (Kgatle, Kalla, Islam, Sathekge, \& Moorad, 2016). The size of the prostate changes with age (ACS, 2016). Pathologic evidence suggests that neoplastic changes of the prostate epithelium develops early in a man's adult life, but do not become clinically evident or problematic until decades later. Some patients live out their lives with prostate cancer that remains stable for decades without treatment. In other cases, cancer grows aggressively, responds poorly to therapy, and causes death within a few years (Sunny, 2005). 
Clinically detected prostate carcinomas display a variety of phenotypic and malignant potential. Majority of all prostate carcinomas are typical adenocarcinomas, which can be divided into different tumour grades. The histological differentiation together with tumour stage, determined by tumour size, as well as the presence of lymph node and distal metastases are used to assess the prognosis of the patients (DF, 1992). Epidemiology studies have shown that there are environmental as well as genetic contributions to the development of prostate cancer. Prostate cancer is a heterogeneous disease, and there is large variations from patient to patient even within the same tumour. These differentials in the Prostate cancer architecture and incidence rates may be attributable to genomic instabilities and alterations associated with various Prostate cancer risk factors.

Cancer of the prostate is primarily a disease of the elderly men. About three-quarters of cases worldwide occur in men aged 40 years and above with increasing incidence and morbidity in men of black African ancestry (Fernandez et al., 2012). In 2012, 1.1 million men were diagnosed with $\mathrm{CaP}$ and $70 \%$ of them (795,000 cases) were in developed countries (Mohammadian-hafshejani, Ghoncheh, Towhidi, Jamehshorani, \& Salehiniya, 2016). Available reports indicate that CaP accounts for approximately $20.3 \%$ and $4.8 \%$ of all male cancers in Sub-Saharan Africa and North Africa, respectively (Cooney, Okuku, \& Orem, 2016; Fernandez et al., 2012). CaP is on the upward trajectory among Nigerian men and remains one of the most common cancer among men in this region (T O Akinremi, Adeniyi, Olutunde, Oduniyi, \& Ogo, 2014). A prevalence rate of 1046 per 100,000 men of age > 40 screened (Ikuerowo et al., 2013) was reported in Lagos. Compared to African-American men, Nigerian men are 10 times more likely to have prostate cancer and 3.5 times more likely to die from it. Environmental and most importantly, genetic factors have been incriminated as the reason for the geographic differences in incidence. Hence, this study seeks to determine the survivorship rate and to compare and contrast the effectiveness of frequentist and Bayesian approaches in modelling the determinants of survivorship among prostate cancer patients. 


\section{Methods}

\section{Study design}

A retrospective analytical study design was employed. This was done through a review and extraction of hospital records, case files of all patients diagnosed and treated for prostate cancer from January 2010 to December 2017 at the University College Hospital (UCH) Ibadan was be included in the study.

\section{Study setting}

The study site includes all patients receiving treatment for prostate cancer at the radiation oncology department of UCH, Ibadan. The department offers services in the area of counselling/psychotherapy, group meeting with patients for problem shearing, alleviation of psychological concern, simulation, tumour localization procedure, palliative care, holistic management for cancer sufferers and their families, External beam radiation therapy, radiotherapy treatment for cancer patients using Cobalt 60 therapy machine, gynecology brachytherapy, ward admission, and clinic services.

\section{Study Population}

This study consists of all Prostate cancer patients who were managed for prostate cancer at the University College Hospital, Ibadan from January 2010 to December 2017.

\section{Data Collection Procedure}

A proforma was used to extract relevant data from patient's records, with the assistance of medical record clerks. The extracted data was divided into two cohorts (2010 - 2014) and (2015 - 2017). A total of 354 patients' records was extracted of which in the (2010 - 2014) cohort, 110 were diseasefree while 54 were censored. Also, in the (2015 - 2017) cohort, 115 patients were disease-free while 75 were censored; with a follow up time of 15 months observed in each cohort. To ensure data reliability, the extracted records was cross-checked by a radiotherapist. The sections in the data collection form included patients' socio-demographics, diagnosis, clinical and pathological features, treatment method, and follow-up. 


\section{Study Variables}

\section{Dependent variable}

The dependent variable was the time to being disease-free (remission) after treatment, measured in months from time of presentation to time patient was last seen. A patient or an individual is declared to be disease-free when there is no residual disease on medical examination after completion of treatment. Meanwhile patients still under follow up, lost to follow up, death from $\mathrm{CaP}$ or other diseases were censored.

\section{Independent variables}

The independent variables for this study include patient's socio-demographics characteristics, clinical characteristics, time of diagnosis, time last seen, time last treated, treatment received and treatment outcome. A description of the coding method is giving in the table below.

\section{Independent variables}

\begin{tabular}{l|l}
\hline Variable & Levels \\
\hline Socio-demographics & \\
Age & $1=50-59,2=60-69,3=70-79,4=79$ - above \\
Marital status & $1=$ married 2=never married 3=widower, 4=divorced \\
Occupation & $1=$ civil servant, 2=farming, 3=factory worker, \\
& $4=$ unemployed, 5=others \\
Religion & $1=$ Christian 2=Islam, 3=Traditional, 4=Others \\
Geographical location & $1=$ south west, 2=south south, 3=south east, 4=north \\
& west, 5=north east, 6=north central \\
Co-morbidities & $1=$ coronary artery disease, 2=diabetes, \\
& $3=$ hypertension/stroke, 4=obesity, 5=psychosocial \\
& stress, 6=alcohol/tobacco, 7=chemical exposure, \\
& $8=$ others \\
Family history of CaP & $1=$ yes, 2=no \\
Clinical/pathological characteristics & 1=PSA test, 2=DRE, 3=Transrectal ultrasound \\
Method of diagnosis & $4=$ biopsy, 5=DRE and biopsy \\
PSA level at diagnosis & ng/ML \\
Stage at diagnosis & $1=$ stage I, 2=stage II, 3=stage III, 4=stage IV \\
Site of metastasis & $5=$ others \\
Gleason group & $1=$ lymph node, 2=rectum (bladder neck), 3=bones, \\
& $4=$ spine, 5=multiple metastasis \\
Treatment & $1=$ well differentiated, 2=moderately differentiated, \\
\hline
\end{tabular}




\begin{tabular}{l|l}
\hline Treatment received & $\begin{array}{l}\text { 1=surgery, 2=radiotherapy, 3=chemotherapy, } \\
\text { 4=radiotherapy and surgery, 5=chemotherapy and } \\
\text { radiotherapy, 6=chemotherapy and surgery, } \\
7=\text { chemotherapy + surgery + radiotherapy } \\
\text { 1=disease-free, 2=death from CaP or other disease, } \\
\text { 3=still under follow up, 4=loss to follow }\end{array}$ \\
Treatment outcome & dd/mm/yy \\
Follow up time & $\mathrm{dd} / \mathrm{mm} / \mathrm{yy}$ \\
Date of diagnosis & $\mathrm{dd} / \mathrm{mm} / \mathrm{yy}$ \\
Date treatment started & $\mathrm{dd} / \mathrm{mm} / \mathrm{yy}$ \\
Date last treated & \\
If loss to follow up, date last seen &
\end{tabular}

\section{Data Management}

Descriptive statistics was used to describe patient's socio-demographic characteristics, clinical characteristics and treatment modality, (frequency, proportions,). Survival probability was determined by Kaplan-Meier survival method. Semi-parametric (Cox proportional hazard) and Bayesian and Frequentist parametric (Weibull) models was used to determine factors affecting survivorship. STATA 15 was employed in the analysis. All analysis was carried out at 5\% significance level.

\section{Determination of survival probabilities}

Due to the presence of right censored data, the Kaplan-Meier method was used in this study.

Suppose we have $\mathrm{p}$ distinct survival times arranged in increasing order, say $t_{1}<t_{2}<\ldots<t_{p}$. At time $t_{i}$ there are $n_{i}$ subjects who are said to be at risk i.e. they survived up to this time and were not censored. The number of subjects who failed at time $t_{i}$ is denoted by $d_{i}$. Consider the recursive equation

$$
\begin{aligned}
S\left(t_{i}\right) & =\mathrm{P}\left(T>t_{i}\right)=\mathrm{P}\left(T>t_{i} \mid T>t_{i-1}\right) * \mathrm{P}\left(T>t_{i-1}\right) \\
& =\mathrm{P}\left(T>t_{i} \mid T>t_{i-1}\right) * S\left(t_{i-1}\right), i=1, \ldots, p
\end{aligned}
$$

Where $t_{0}=0$ and $S_{0}=1$

Therefore, the survival function is given as

$$
s(t)=S^{\prime}(t)=\frac{d}{d t} S(t)=\frac{d}{d t} \int_{t}^{\infty} f(u) d u=\frac{d}{d t}[1-F(t)]=-f(t)
$$


The survival time $t$ is the time period (months) to being disease-free from time of diagnosis to time last treated/seen, since all patients are at risk of this event. The censoring index for patients who were disease-free is " 1 " whereas patients who are is still undergoing treatment, died from CaP or a disease other than $\mathrm{CaP}$, patients who can no longer be observed (Loss-to-follow-up), are declared censored and coded as " 0 ”. While the log-rank test was used to test the equality of survival curves.

\section{Cox Proportional hazard model}

As a nod to the proportional hazard assumption, consider two subjects for which the values of the covariates are $x_{i 1}, \ldots, x_{i p}$ and $x_{j 1}, \ldots, x_{j p}$, respectively. The ratio of the hazard function is

$$
\frac{h\left(t, x_{i 1}, \ldots, x_{i p}, \beta_{1}, \ldots, \beta_{p}\right)}{h\left(t, x_{j 1}, \ldots, x_{j p}, \beta_{1}, \ldots, \beta_{p}\right)}=\exp \left\{\beta_{1}\left(x_{i 1}-x_{j 1}\right)+\cdots+\beta_{p}\left(x_{i p}-x_{j p}\right)\right\}
$$

Given the risk of death at a particular time, depends on the values $x_{1}, x_{2}, \ldots, x_{p}$ of $p$ explanatory variables, $X_{1}, X_{2}, \ldots, X_{p}$. These values will be assumed to have been recorded at the time origin of the study; assuming the explanatory variables do not depend on time, the hazard function has the following form:

$$
h\left(t, x_{1}, \ldots, x_{p}, \beta_{1}, \ldots, \beta_{p}\right)=h_{0}(t) \exp \left\{\beta_{1} x_{1}+\cdots+\beta_{p} x_{p}\right\}
$$

The function $h_{0}(t)$ is called the baseline hazard function

For this study, the Proportion hazard model was fitted for the following independent variables (age, occupation, marital status, methods of diagnosis, Gleason group, PSA level, Stage at diagnosis, site of metastasis, treatment type). The coefficients of the independent variables were estimated using partial likelihood method, using the "stcox" package in STATA 15.

i.e. $\log \left(\frac{h_{i}(t)}{h_{0}(t)}\right)=B_{1} X_{1}+B_{2} X_{2 i}+B_{3} X_{3 i}+B_{4} X_{4 i}+B_{5} X_{5 i}+B_{6} X_{6 i}+B_{7} X_{7 i}+B_{8} X_{8 i}+B_{9} X_{9 i}$

Where

$X_{1}=$ Age, $X_{2 i}=$ Occupation, $X_{3 i}=$ Marital Status, $X_{4 i}=$ Method of diagnosis

$X_{5 i}=$ Grade of disease, $X_{6 i}=$ PSA level at diagnosis, $X_{7 i}=$ Stage at diagnosis

$X_{8 i}=$ Site of metastasis, $X_{9 i}=$ Treatment modality

\section{The Weibull Accelerated Failure time (AFT) Model (Frequentist)}

Accelerated failure time models, also known as accelerated-time models or In(time) models follow the parameterization 


$$
\ln \left(t_{j}\right)=x_{j} \beta_{X}+\epsilon_{J}
$$

The word "accelerated" is used in describing these models because rather than assuming that failure time $t_{j}$ is exponential, Weibull, or some other form, a distribution is instead assumed for

$$
\tau_{j}=\exp \left(-x_{j} \beta_{X}\right) t_{j}
$$

and $\exp \left(-x_{j} \beta_{X}\right)$ is called the acceleration parameter

Assuming that the survival time follows a Weibull distribution with scale parameter $\beta_{0}$ and shape parameter $p$, written as

$$
\tau_{j} \sim \operatorname{Weibull}\left(\beta_{0}, p\right)
$$

$\tau_{j}$ is distributed as Weibull with parameters $\left(\beta_{0}, p\right)$.

The Weibull hazard and survivor functions are

$$
h_{0}(t)=\beta_{0} p t^{p-1}
$$

and

$$
S_{i}(t)=\exp \left(-\beta_{0} t^{p}\right)
$$

respectively,

Since the effect of the covariates is to accelerate time by a factor of $\exp \left(-x_{j} \beta_{X}\right)$, thus for the AFT model

$$
\begin{aligned}
& S\left(t_{j} \mid x_{j}\right)=S_{0}\left\{\exp \left(-x_{j} \beta_{X}\right) t_{j}\right\} \\
= & \exp \left[-\left\{\exp \left(-\beta_{0}\right) \exp \left(-x_{j} \beta_{X}\right) t_{j}\right\}^{p}\right] \\
= & \exp \left[-\left\{\exp \left(-\beta_{0}-x_{j} \beta_{X} t_{j}\right\}^{p}\right]\right.
\end{aligned}
$$

The "streg" package in STATA 15 was used to estimate the maximum likelihood estimate for the Weibull AFT model by specifying the "tr(time ratio)" option. Essentially, the accelerated failure time implies a deceleration of time or, in other terms, an increment in the expected waiting time for an event of interest to occur. Time Ratio (TR) $>1$ indicates that an individual experience being disease-free at an earlier timing, $\mathrm{TR}=1$ implies no difference in survival time, while $\mathrm{TR}<1$ suggests that an individual experienced a delay or decreased in time to being disease-free.

\section{Bayesian Survival Analysis}

Bayesian analysis generates conclusions based on the synthesis of new information from the observed data and previous knowledge or external evidence. The Weibull Model is one of the 
accelerated failure time models that can be used fit survival data under the Bayesian framework. Let $t_{i}$ denote the survival time of the $i^{\text {th }}$ patient. Assuming that $t_{i}$ has a Weibull distribution with parameters $\alpha>0$ and $r>0$, with a density function of the form:

$$
f\left(t_{i} \mid \alpha, r\right)=\alpha t_{i}^{\alpha-1} \exp -\left[r t_{i}\right] \alpha, \quad 0<t_{i}<\infty
$$

The survival function of $t_{i}$ is given by

$$
S\left(t_{i} \mid \alpha, r\right)=\exp \left(-\exp \left(r t_{i}\right) t_{i} \alpha\right)
$$

The hazard function is given by

$$
\left.h\left(t_{i} \mid \alpha, r\right)=r \alpha t_{i}^{\alpha-1}\right)
$$

The likelihood function of the unknown parameters $(\alpha, r)$ given the data can be written as:

$$
\begin{array}{r}
L(\alpha, r \mid D)=\prod_{i=1}^{n} f\left(t_{i} \mid \alpha, r\right) S\left(t_{i} \mid \alpha, r\right)^{\left(1-\delta_{i}\right)} \\
\left.=a \sum \delta_{i} \exp \left\{r \sum_{i=1}^{n} \delta_{i}+\sum_{i=1}^{n} \delta_{i}(a-1) \log \left(t_{i}\right)-\exp (r) t_{i} \alpha\right)\right\}
\end{array}
$$

Where $\delta_{i}$ is an indicator variable taking value 1 if $t_{i}$ is the failure time (i.e patients who are diseasefree) and 0 if $t_{i}$ is right censored (loss-follow-up, death, still under follow up).

To incorporate covariates we, therefore, write $r=X^{\prime}{ }_{i} \beta$, where $X^{\prime}{ }_{i}$ and $\beta$ are $p x 1$ vector of covariates and regression coefficients respectively.

Assuming a normal prior for $\beta, N_{p}\left(\mu_{0}, \sum_{0}\right)$ then the joint posterior is given by

$$
\begin{gathered}
\prod(\beta, \alpha \mid D)^{\alpha} \alpha^{\alpha_{0}+d+1} \exp \sum_{i=1}^{n}\left(\delta_{i}+\boldsymbol{X}^{\prime}{ }_{i} \beta+\delta_{i}(\alpha-1) \log \left(t_{i}\right)\right)-t_{i}^{\alpha} \exp \left(\boldsymbol{X}^{\prime}{ }_{i} \beta\right)-k_{0}^{\alpha}-\frac{1}{2}(\beta \\
\left.-\mu_{0}\right) \Sigma_{0}^{-1}\left(\beta-\mu_{0}\right)
\end{gathered}
$$

Where $\mathrm{D}=(n, t, X, \delta)$ denote the observed data for the regression model and $X$ is an $n x p$ matrix of covariates with the $i$-th row as $X^{\prime}{ }_{i}$ and $\delta=\left(\delta_{i}, \ldots, \delta_{n}\right)^{\prime}$.

The "bayes, streg, tr" package in STATA 15 was used to fit the Bayesian AFT model using the random-walk Metropolis-Hastings Monte Carlo Markov Chain (MCMC) sampling method for simulating the results of the parameters . The Metropolis-Hastings algorithm is given as follows: Let $q(\theta, \vartheta)$ be a proposal density which is also termed as a candidate generating density such that

$$
\int q(\theta, \vartheta) d \vartheta=1
$$


Also, let $\mu(0,1)$ denote the uniform distribution over $(0,1)$. Then, a general version of the Metropolis-Hastings algorithm for sampling from the posterior distribution $P(\boldsymbol{\theta} \mid \boldsymbol{x})$ can be described as follows:

Step 0. Choose an arbitrary starting point $\theta_{0}$ and set $i=0$.

Step 1.Generate a candidate point $\theta^{*}$ from $q\left(\theta_{i},.\right)$ and $u$ from $\mu(0,1)$

Step 2. Set $\theta_{i+1}=\theta^{*}$ if $u \leq a\left(\theta_{i}, \theta^{*}\right)$ and $\theta_{i+1}=\theta_{i}$ otherwise, where the acceptance probability is given by

$$
a(\theta, \vartheta)=\min \left\{\frac{\pi(\vartheta \mid D) q(\theta, \vartheta)}{\pi(\vartheta \mid D) q(\theta, \vartheta)}, 1\right\}
$$

Step 3. Set $i=i+1$, and go to Step 1 .

The iteration steps from 0 through 3 is referred to as an MH update. By design, any Markov chain simulated using this $\mathrm{MH}$ algorithm is guaranteed to have the posterior distribution as its stationary distribution.

Criteria's for measuring the efficiency of MCMC are the acceptance rate of the chain and the degree of autocorrelation in the generated sample. When the acceptance rate is close to " 0 " then most of the proposals are rejected, which means that the chain failed to explore regions of appreciable posterior probability. The cut-off for acceptance rate is 0.234 for a multivariate posterior and 0.45 for a univariate posterior.

\section{Model Evaluation Methods}

The model was evaluated using -2LogL, Akaike Information Criterion (AIC) Bayesian Information Criterion (BIC) Deviance Information Criterion (DIC). Among the (2010 - 2014), (2015 - 2017) cohorts model and overall group, any of Cox Proportional Hazard (CPH), parametric regression model with consistent low values of $-2 L L$, AIC and BIC was selected as the best fitting model while for Bayesian regression a model with consistently low values of -2LL, AIC, BIC, DIC and an acceptance rate of 0.2 for the multivariate model and 0.4 for the univariate model was selected as the best fitting model.

\section{Ethical consideration}

Ethical approval was obtained from UI/UCH Ethics Committee of the University of Ibadan approval reference: UI/EC/18/0492 


\section{Results}

\section{Distribution of prostate cancer $(\mathrm{CaP})$ patients by sociodemographic characteristics}

Three hundred and fifty-four patients were observed age 50 - 104 years, with an average age of 72 years and a standard deviation of 9.52. Patients 50 - 59 years were 33(9.3\%), 60 - 69 years 90(25.4\%) The peak incidence of CaP was observed among those age 70 - 79 years 141(39.8\%) while those 79 years above were $90(25.4 \%)$. Those married were $347(97.7 \%)$ while $8(2.3 \%)$ were widowers. 181(51.1\%) civil servants, $4(1.1 \%)$ unemployed, $9(2.5 \%)$ farmers and $16(4.5 \%)$ factory workers and others (taxi drivers, artisans, labourers, business men) 144(40.7\%).

Of the 354 cases, 257(72.6\%) were Christians, 94(26.6\%) Islam while 3(0.8\%) practised other religion, 278(78.5\%) were from south-western Nigeria 38(10.7\%) south-south, 30(8.5\%) southeast while $8(2.3 \%)$ from north central. Those with a family history of prostate cancer were $77(21.8 \%)$ while $167(33.0 \%)$ smoked tobacco or drank alcohol, 101(20.0\%) had coronary heart disease, 118(23.3\%) diabetes, and 120(23.7\%) were hypertensive (table 1).

Table 1: Distribution of prostate cancer patients by sociodemographic characteristics

\begin{tabular}{lcc}
\hline Characteristics & n & \% \\
\hline Age (years) & 33 & 9.3 \\
$50-59$ & 90 & 25.4 \\
$60-69$ & 141 & 39.8 \\
$70-79$ & 90 & 25.4 \\
$79-$ above & & \\
Marital Status & 346 & 97.7 \\
Currently married & 8 & 2.3 \\
Widower & & \\
Occupation & 181 & 51.1 \\
Civil Servant & 9 & 2.5 \\
Farming & 16 & 4.5 \\
Factory Worker & 4 & 1.1 \\
Unemployed & 144 & 40.7 \\
Others & & \\
Religion & 257 & 72.6 \\
Christian & 94 & 26.6 \\
Islam & 3 & 0.8 \\
Others & & \\
Geographical Location & 278 & 78.5
\end{tabular}


South-south

South-east

Family History of Prostate Cancer

Yes

No

Year of Diagnosis

2010

45

78.2

2011

18

12.7

2012

2013

4

2014

26

5.1

2015

2016

64

59

7.3

2017

67

20.1

18.1

16.7

18.9

Co-morbidities

Alcohol and Tobacco

167

33.0

Coronary Artery

101

20.0

Diabetes

118

23.3

Hypertension/Stroke

120

23.7

Total

354

100.0

Others include: taxi drivers, artisans, labourers, business men

\section{Distribution of CaP patients by clinical characteristics}

Of the 354 Patients enrolled into this study, 193(54.5\%) were diagnosed via Digital Rectal Examination (DRE), Biopsy 98(27.7\%), Prostate Specific Antigen test 20(5.6\%), while 34(9.6\%) using both DRE and Biopsy. Patients with a poorly differentiated Gleason score were 220(62.1\%), 62(17.5\%) were moderately differentiated while $72(20.3 \%)$ were well differentiated; 309(87.3\%) were diagnosed at stage IV while 11(3.1\%) were diagnosed at stage I, 18(5.1\%) had metastasis to the lymph node, 55(15.5\%) rectum, $84(23.7 \%)$ bones, $116(32.8 \%)$ spine, while about a quarter had multiple metastases $81(22.9 \%)$.

Surgery was carried out on 67(18.9\%) patients, while 42(11.9\%) and 62(17.5\%) received radiotherapy and chemotherapy respectively, combination therapy was applied to $7(2.0 \%)$, radiotherapy and surgery 48(13.6\%), 48(13.6\%) chemotherapy and radiotherapy, 119(33.6\%) Chemotherapy and surgery while $9(2.5 \%)$ received all the three combinations of chemotherapy, surgery and radiotherapy. Of the total patients record extracted during this period, 225(55.6\%) 
were disease-free after treatment, 17(10.2\%) died from prostate cancer, 75(25.0\%) were still under follow-up while 37(9.2\%) were lost to follow-up (table 2).

Table 2: Distribution of prostate cancer patients by clinical characteristics

\begin{tabular}{lcc}
\hline Characteristics & $\mathbf{n}$ & \% \\
\hline Method of Diagnosis & 20 & 5.6 \\
PSA test & 193 & 54.5 \\
DRE test & 9 & 2.5 \\
Transrectal Ultrasound & 98 & 27.7 \\
Biopsy & 34 & 9.6
\end{tabular}

Gleason group

Well-differentiated $\quad 72$

20.3

Moderately differentiated

Poorly differentiated

\section{Stage at Diagnosis}

Stage I

Stage II

Stage III

Stage IV

\section{Site of Metastasis}

Lymph node

Rectum (bladder neck)

Bones

Spine

Multiple Metastasis
Treatment Modality

Surgery alone

Radiotherapy alone

Chemotherapy alone

Radiotherapy \& Surgery

Chemotherapy \& Radiotherapy

Chemotherapy \& Surgery

Chemotherapy \& Surgery \& Radiotherapy
5.1

15.5

23.7

32.8

22.9

18.9

11.9

17.5

2.0

13.6

33.6

2.5

55.6

225

10.2

75 
Figure 1: Distribution of CaP patients by stage at diagnosis and site of metastasis

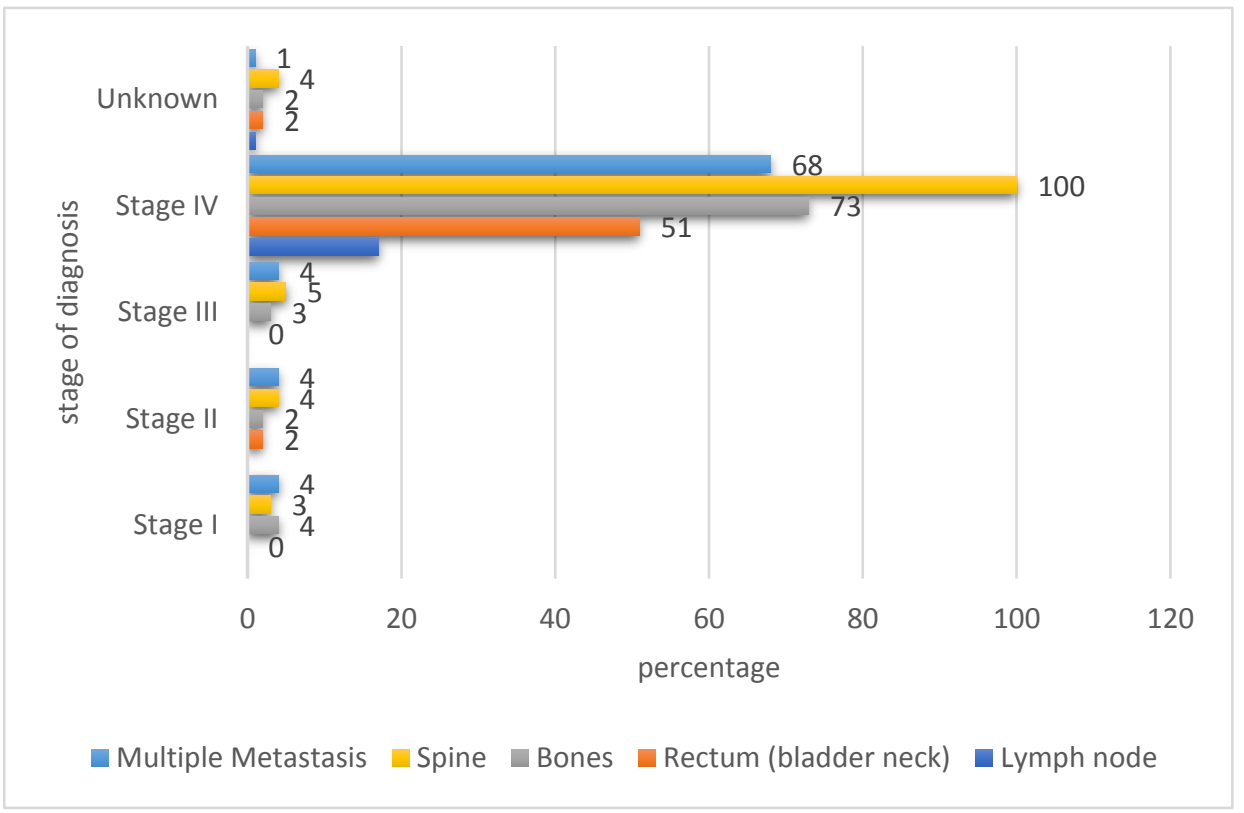

\section{Median survival time of socio-demographics and clinical characteristics among CaP patients}

The result showed that a total of 354 patients were diagnosed and treated for CaP between 2010 2017, with a follow up time of 15 months observed for each patient, of which for the 164 patients in the 2010 - 2014 cohort, 110 were disease-free while 54 were censored. Among the 190 patients in the 2015 - 2017 cohort; 115 were disease-free while 75 were censored. It was observed that patients aged 50 - 59 years in the 2010 - 2014 cohort had a MS time of 3.5 months while those in the 2015 - 2017 cohort had a MS time of 3.8 months, patients $60-69$ years in the $2010-2014$ cohort had a MS time of 4.2 months; meanwhile patients age 79 years and above had a median survival time of 2.2 months in the 2010 - 2014 cohort (table 3 ).

Those who were married, had a MS time of 2.8 months among the $2010-2014$ cohort and 3.3 months among the 2015 - 2017 cohort respectively and an overall median survival time of 3.2 months, while widowers had a median survival time of less than one month among patents in the 2010 - 2014 cohort and 0.9 months among the 2015 - 2017 cohort. Patients from the south-western 
part of the country had a MS time of almost three months for the 2010 - 2014 cohort and more than three months for the 2015 - 2017 cohort of patients, while patients from the south-south region had a MS time of 1.5 months for the 2010 - 2014 cohort of patients and more than four and a half months among the 2015 - 2017 cohort; overall MS time was 2.6 months. The highest MS time was recorded amongst those from the north central region 14.2 months in the $2015-2017$ cohort (table 3).

The MS time of patients with a family history of CaP among the 2010 - 2014 cohort of patients was three and a half months, and an overall MS time of 2.8 months while those with no family of CaP had a MS time of 2.9 months for the 2010 - 2014 cohort of patients and 3.5 months among the 2015 - 2017 cohort. Overall MS time was 3.1 months for those with no family history of prostate cancer (table 3 ).

MS time among patients diagnosed via Prostate Specific Antigen (PSA) test was 3.1 months among the 2010 - 2014 cohort and 2.8 months for the 2015 - 2017 cohort while those diagnosed using Digital Rectal Examination (DRE) had a MS time of 2.9 months for the 2010 - 2014 cohort of patients and more than three and a half month for the 2015 - 2017 cohort with an overall MS time of three and a half month, similarly patients diagnosed with a combination of Biopsy and DRE had a MS time of a little above two and half months for the 2010 - 2014 cohort of patients and more than four months for the 2015 - 2017 cohort. Patients whose Gleason group were categorized as well differentiated had a median survival time of 1.9 months for the 2010 - 2014 cohort of patients and 4.7 months for the 2015 - 2017 cohort (table 3); while those poorly differentiated had a MS time of 3.5 months for the 2010 - 2014 cohort and 3.3 months for the 2015 - 2017 cohort of patients. Probability of being disease-free was highest among those diagnosed via DRE and Biopsy in the 2015 - 2017 cohort, while patients with a well differentiated Gleason score also had a high probability of being disease-free (figure 3).

Of the 164 patients; patients in the 2010 - 2014 cohort, those diagnosed at stage I had a MS time of 2.4 months, while among the 2015 - 2017 cohort of patients was 3.0 months; those diagnosed at stage IV had a MS time of 3.1 months for the 2010 - 2014 cohort and 3.4 months for the 2015 - 2017 cohort of patients. The lowest MS time was recorded among those diagnosed at stage III, 
1.4 months among the 2010 - 2014 cohort of patients. Meanwhile among the 190 patients in the 2015 - 2017 cohort, those diagnosed at stage II had a MS time of three and a half months; patients with lymph node metastasis had a MS time of four months with an overall MS time of 3.5 months, as those with metastasis to the rectum. Patients with metastasis to the bones had a MS time of 2.4 months among the $2010-2014$ cohort of patients and 2.9 months for patients in the $2015-2017$ cohort. Those with multiple metastases in the 2010 - 2014 cohort survived for a period of 4.2 months while those in the 2015 - 2017 cohort survived for three months; overall survival time for multiple metastasis was 3.4 months. Probability of being disease-free was lowest among those diagnosed at stage IV in the 2010 - 2014 cohort while it was observed to be highest in the 2015 2017 cohort (figure 3).

Patients who underwent surgery among the 2010 - 2014 cohort survived for a period of 1.6 months and 2.4 for those in 2015 - 2017 cohort. Overall MS time was 2.2 months, while those who received radiotherapy alone had a MS time of one and a half month. Those treated with combination of radiotherapy and surgery had a MS time of 4.6 months while those treated with chemotherapy and surgery had a MS time of more than five months in the 2010 - 2014 cohort. Among patients in the 2015 - 2017 cohort, those treated with radiotherapy and surgery survived for 6.9 months and 4.6 months for those in the 2010 - 2014 cohort, with an overall MS time of 6.9 months, those treated with chemotherapy and radiotherapy survived for 4.0 months, with an overall MS time of 3.7 months. Patients treated with chemotherapy and surgery survived for almost four and a half months in the 2015 - 2017 cohort, and 5.9 months among those in the $2010-2014$ cohort, with an overall survival time of 4.6 months.

Probability of being disease-free was highest among those who were treated with radiotherapy and surgery in the 2015 - 2017 cohort, while it was lowest for those treated with radiotherapy alone in the 2010 - 2014 cohort of patients (Figure 3) The overall MS time for the 2015 - 2017 cohort of patients was 3.3 months meanwhile for the 2010 - 2014 cohort the MS time was 2.9 months (table 3). As shown in Figure 2, patients in the 2015 - 2017 cohort had the highest probability of being disease-free compared to patients in the 2010 - 2014 cohort. The overall MS time for the study was 3.2 months (table 3). 
Table 3: Median survival time of prostate cancer patients

\begin{tabular}{|c|c|c|c|c|c|c|}
\hline \multirow[t]{2}{*}{ Characteristic } & \multicolumn{2}{|c|}{$2010-2014$} & \multicolumn{2}{|c|}{$2015-2017$} & \multicolumn{2}{|c|}{ Overall } \\
\hline & n & MST & $\mathbf{n}$ & MST & $\mathbf{n}$ & MST \\
\hline Age & & & & & & \\
\hline $50-59$ & 15 & 3.5 & 18 & 3.8 & 33 & 3.5 \\
\hline $60-69$ & 44 & 4.2 & 46 & 2.9 & 90 & 3.6 \\
\hline $70-79$ & 69 & 2.7 & 72 & 3.4 & 141 & 2.9 \\
\hline 79 - above & 36 & 2.2 & 54 & 3.4 & 90 & 2.8 \\
\hline \multicolumn{7}{|l|}{ Marital Status } \\
\hline Currently married & 160 & 2.8 & 186 & 3.3 & 346 & 3.2 \\
\hline Widower & 4 & 0.7 & 4 & 0.9 & 8 & 0.9 \\
\hline \multicolumn{7}{|l|}{ Occupation } \\
\hline Civil Servant & 92 & 2.6 & 89 & 3.0 & 181 & 2.9 \\
\hline Farming & 3 & 1.8 & 6 & 2.8 & 9 & 2.8 \\
\hline Factory Worker & 9 & 5.2 & 7 & 4.5 & 16 & 5.1 \\
\hline Unemployed & 3 & 1.1 & 1 & $\mathrm{CBC}$ & 4 & 1.2 \\
\hline Others & 57 & 3.5 & 87 & 3.3 & 144 & 3.3 \\
\hline \multicolumn{7}{|l|}{ Religion } \\
\hline Christian & 121 & 2.9 & 136 & 3.3 & 257 & 3.2 \\
\hline Islam & 41 & 2.5 & 53 & 3.4 & 94 & 2.7 \\
\hline Others & 2 & 1.8 & 1 & $\mathrm{CBC}$ & 3 & 3.7 \\
\hline \multicolumn{7}{|l|}{ Geographical Location } \\
\hline South-west & 132 & 2.9 & 146 & 3.3 & 278 & 3.1 \\
\hline South-south & 18 & 1.5 & 20 & 4.9 & 38 & 2.6 \\
\hline South-east & 10 & 3.5 & 20 & 3.3 & 30 & 3.3 \\
\hline North-central & 4 & 2.5 & 4 & 14.2 & 8 & 3.7 \\
\hline \multicolumn{7}{|c|}{ Family History of Prostate Cancer } \\
\hline Yes & 35 & 3.5 & 42 & 2.4 & 77 & 2.8 \\
\hline No & 129 & 2.9 & 148 & 3.5 & 277 & 3.1 \\
\hline \multicolumn{7}{|l|}{ Method of diagnosis } \\
\hline PSA test & 10 & 3.1 & 10 & 2.8 & 20 & 2.9 \\
\hline DRE test & 90 & 2.9 & 103 & 3.8 & 193 & 3.5 \\
\hline Transrectal Ultrasound & 5 & 2.3 & 4 & 0.6 & 9 & 1.4 \\
\hline Biopsy & 44 & 2.9 & 54 & 2.9 & 98 & 2.9 \\
\hline DRE \& Biopsy & 15 & 2.6 & 19 & 4.2 & 34 & 2.7 \\
\hline \multicolumn{7}{|l|}{ Gleason group } \\
\hline Well differentiated & 32 & 1.9 & 40 & 4.7 & 72 & 2.9 \\
\hline Moderately differentiated & 32 & 3.2 & 30 & 3.0 & 62 & 3.0 \\
\hline Poorly differentiated & 100 & 3.5 & 120 & 3.3 & 220 & 3.3 \\
\hline
\end{tabular}


Stage at Diagnosis

Stage I

Stage II

Stage III

Stage IV

Unknown

Site of Metastasis

Lymph node

Rectum (bladder neck)

Bones

Spine

Multiple Metastasis

Treatment Modality

Surgery alone

Radiotherapy alone

Chemotherapy alone

Radiotherapy \& Surgery

Chemotherapy \& Radiotherapy

Chemotherapy \& Surgery

Chemotherapy \& Surgery \& Radiotherapy

Overall

NB: $C B C=$ can't be computed; Overall $=2010-2017$

\begin{tabular}{c|c|c|c|c|c} 
& & & & & \\
6 & & & & & \\
6 & 2.4 & 5 & 3.0 & 11 & 2.3 \\
8 & 2.1 & 6 & 3.5 & 12 & 3.3 \\
137 & 1.4 & 4 & 1.6 & 12 & 1.6 \\
7 & 3.1 & 172 & 3.4 & 309 & 3.3 \\
& 3.5 & 3 & 1.5 & 10 & 3.5 \\
& & & & & \\
6 & & & & & \\
22 & 3.1 & 33 & 4.0 & 55 & 3.5 \\
41 & 2.4 & 43 & 2.9 & 84 & 2.7 \\
56 & 2.6 & 60 & 3.3 & 116 & 2.9 \\
39 & 4.2 & 42 & 3.0 & 81 & 3.4 \\
& & & & & \\
29 & 1.6 & 38 & 2.4 & 67 & 2.2 \\
22 & 1.5 & 20 & 3.2 & 42 & 2.6 \\
28 & 2.9 & 34 & 2.7 & 62 & 2.9 \\
3 & 4.6 & 4 & 6.9 & 7 & 6.9 \\
24 & 1.7 & 24 & 4.0 & 48 & 3.7 \\
56 & 5.9 & 63 & 4.4 & 119 & 4.6 \\
2 & 1.9 & 7 & $\mathrm{CBC}$ & 9 & $\mathrm{CBC}$ \\
164 & 2.9 & 190 & 3.3 & 354 & 3.2 \\
\hline 2017 & & & & &
\end{tabular}

Figure 2: Survivorship curves showing the probability of survival for cohort $2010-2014$ and

$2015-2017$ 


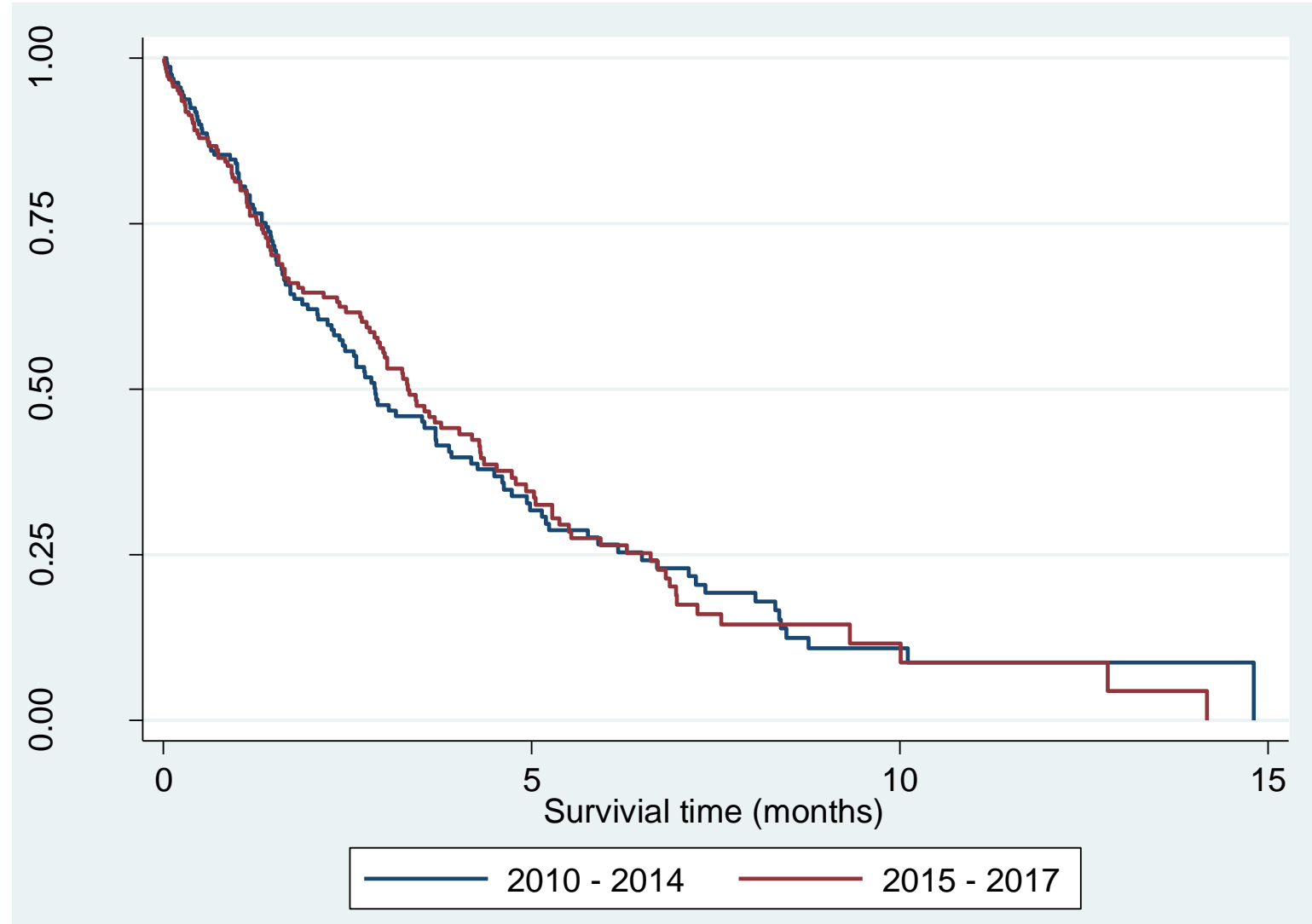

Comparison of survivor function across the selected Sociodemographic and clinical characteristics of the CaP patients

The comparison of survivor curves showed that the probability of survival was significantly different, across the different patients' age, for those in the 2010 - 2014 and 2015 - 2017 cohorts respectively. There were no differences in survival experiences among patients with characteristics such as occupation, family history, methods of diagnosis, Gleason group, stage at diagnosis, site of metastasis across 2010 - 2014 and 2015 - 2017 cohort.

Treatment received showed a significant difference in survivorship experience for patients in the 2010 - 2014 and 2015 - 2017 cohort. Overall treatment regimen received showed significant difference in survival experience for all patients (Table 4).

Table 4: Comparison of survivor function across selected demographic and clinical characteristics of CaP Patients 


\begin{tabular}{|c|c|c|c|}
\hline & 2010 - 2014 & $2015-2017$ & Overall \\
\hline Characteristics & $\chi^{2}, P$-value $*$ & $\chi^{2}, P$-value ${ }^{*}$ & $\chi^{2}, P$-value ${ }^{*}$ \\
\hline Age & $61.55,<\mathbf{0 . 0 5}$ & $55.28,<\mathbf{0 . 0 5}$ & $0.56,0.75$ \\
\hline Occupation & $1.72,0.78$ & $4.59,0.33$ & $4.37,0.36$ \\
\hline Family History & $0.92,0.33$ & $3.23,0.07$ & $0.22,0.64$ \\
\hline Method of diagnosis & $2.39,0.66$ & $3.13,0.53$ & $3.41,0.49$ \\
\hline Gleason group & $2.58,0.27$ & $4.79,0.09$ & $1.13,0.57$ \\
\hline Stage at Diagnosis & $3.07,0.54$ & $3.02,0.55$ & $5.58,0.23$ \\
\hline Site of Metastasis & $0.66,0.96$ & $0.19,0.99$ & $0.39,0.98$ \\
\hline Treatment Modality & $22.57,<\mathbf{0 . 0 5}$ & $14.22,<\mathbf{0 . 0 3}$ & $24.87,<\mathbf{0 . 0 5}$ \\
\hline
\end{tabular}

Overall $=2010-2017 *$ from Log rank test 


\section{Figure 3: Kaplan Meier survival curves for selected clinical characteristics}

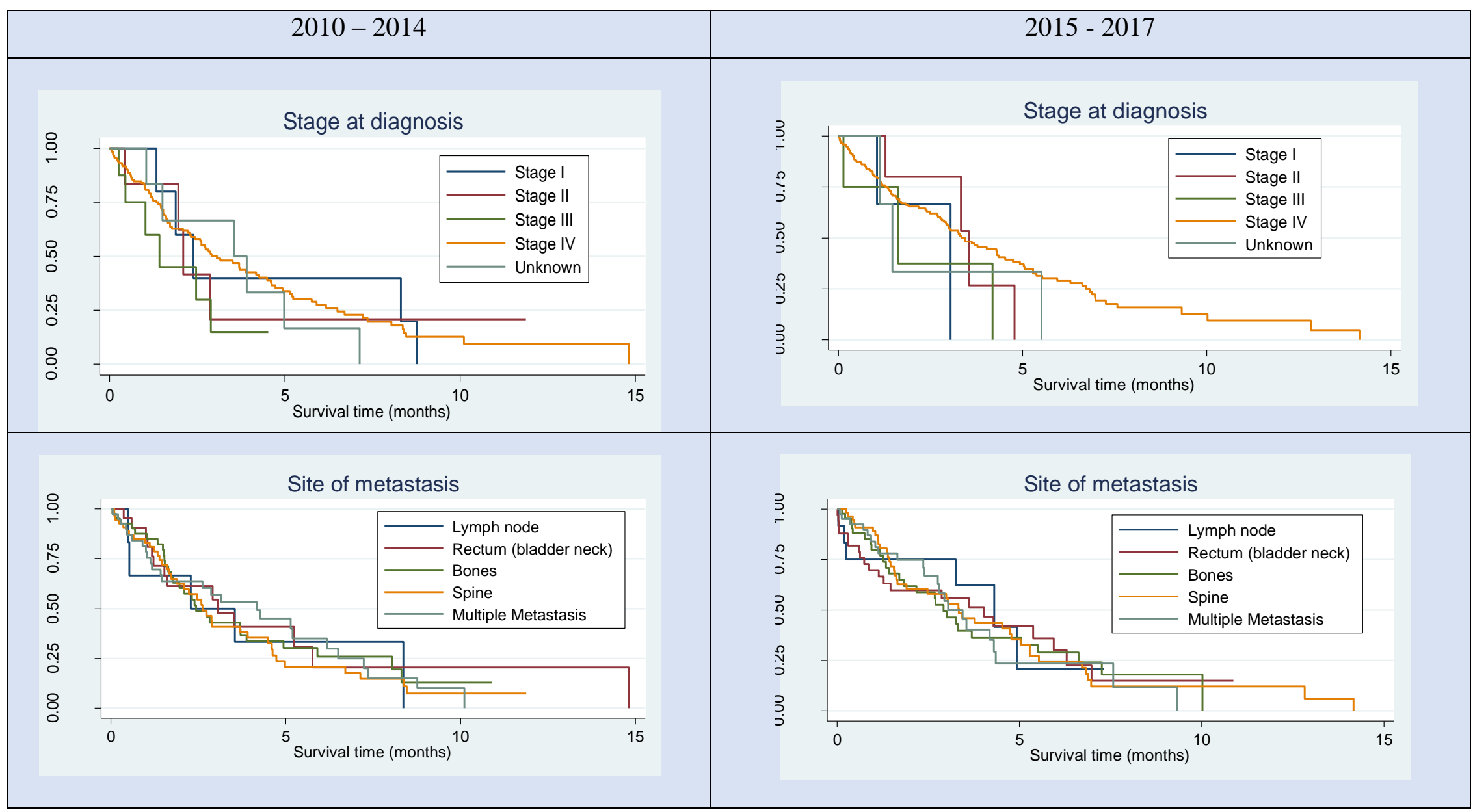




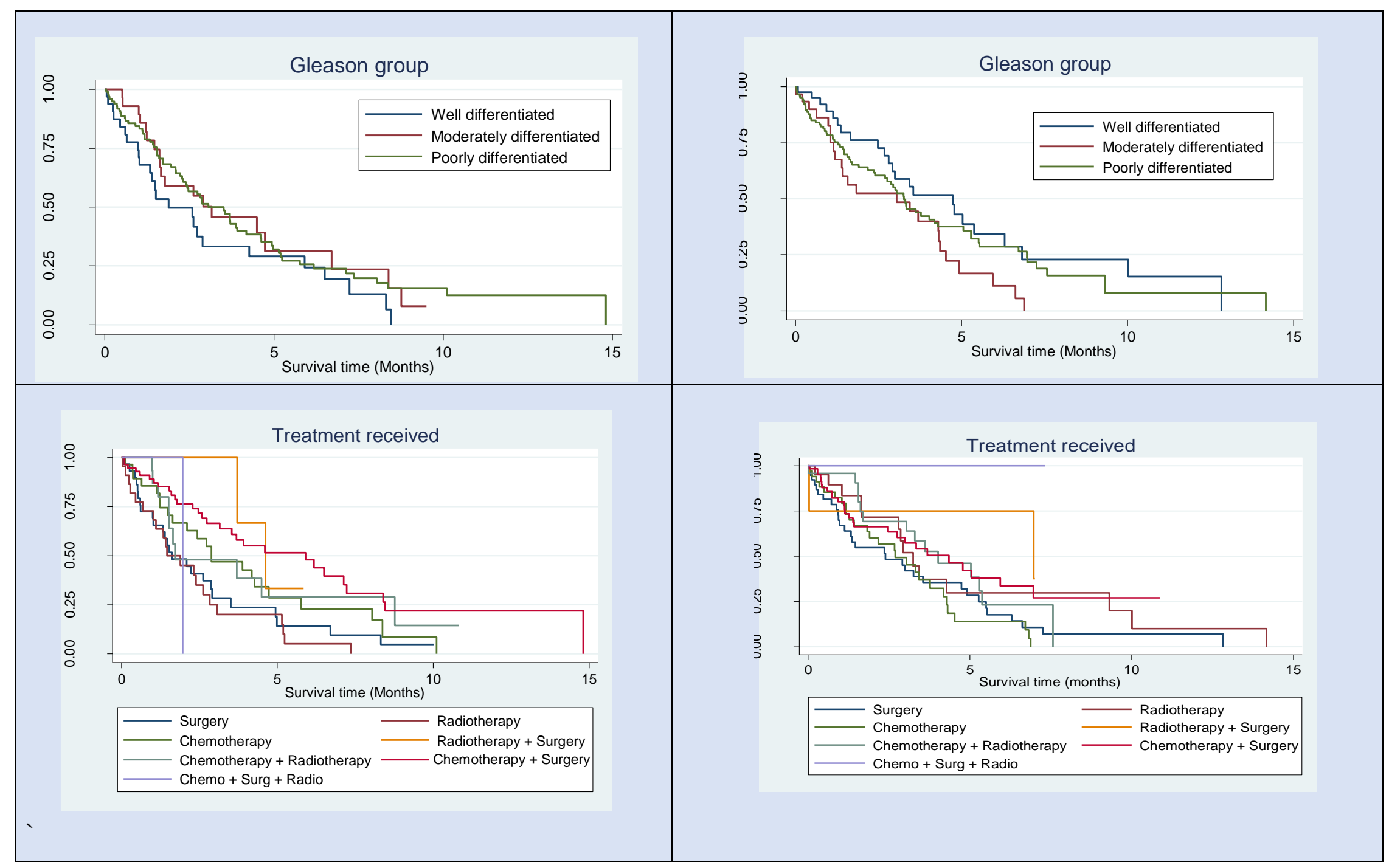




\section{Cox proportional regression}

The outcome of the bivariate regression models showed that widowers in the $2015-2017$ cohort had a $30 \%$ increase in risk $(\mathrm{HR}=3.3$; 95\% CI: $1.0-10.5)$ of being disease-free compared to patients who were currently married (Table 4).

Patients treated using a combination of chemotherapy and surgery experienced a $60 \%,(\mathrm{HR}=0.6$; 95\%CI: $0.4-1.0)$ and 40\% (HR $=0.4$; 95\%CI: $0.2-0.7)$ delay in risk of being disease-free for the 2015 - 2017, and 2010 - 2014 cohorts respectively. Meanwhile, patients who received a combination of chemotherapy, surgery and radiotherapy in the overall group had a 10\% delay (HR $=0.1 ; 95 \% \mathrm{CI}: 0.1-0.9)$ in risk of being disease-free compared to patients who underwent surgery alone.

Table 4: Unadjusted determinants of prostate cancer survivorship using Cox proportional hazard regression model

\begin{tabular}{|c|c|c|c|}
\hline Characteristic & 2010 - 2014 & 2015 - 2017 & Overall \\
\hline & HR(95\% CI) & HR(95\%CI) & HR(95\% CI) \\
\hline \multicolumn{4}{|l|}{ Age } \\
\hline $50-59$ & 1.0 & 1.0 & 1.0 \\
\hline $60-69$ & $1.1(0.5-2.3)$ & $0.9(0.5-2.1)$ & $1.0(0.6-1.7)$ \\
\hline $70-79$ & $1.1(0.5-2.2)$ & $1.1(0.5-2.1)$ & $1.0(0.6-1.7)$ \\
\hline 79 - above & $1.4(0.7-3.1)$ & $1.0(0.5-2.0)$ & $1.1(0.7-1.9)$ \\
\hline
\end{tabular}

\section{Marital Status}

Currently married

1.0

1.0

1.0

Widower

$$
1.9(0.6-6.1) \quad 3.3(1.0-10.5)^{*} \quad 2.5(1.0-5.6)^{*}
$$

\section{Occupation}

Civil Servant

Farming

1.0

1.0

1.0

Factory Worker

$1.2(0.3-5.2)$

$1.3(0.5-3.1)$

$1.3(0.6-2.7)$

$0.6(0.3-1.4)$

$0.3(0.1-1.4)$

$0.5(0.2-1.0)$

Unemployed

$1.2(0.3-5.0)$

$0.6(0.1-4.3)$

$0.9(0.3-2.8)$

Others

$1.0(0.7-1.5)$

$0.8(0.5-1.1)$

$0.9(0.7-1.2)$

\section{Religion}

Christian

1.0

Islam

Others
$1.5(1.0-2.4)$

$1.7(0.4-7.1)$
1.0

$0.9(0.6-1.3)$

$\mathrm{CBC}$
1.0

$1.2(0.9-1.5)$

$0.9(0.2-3.8)$

Geographical Location

South-west

1.0

1.0

1.0 
South-south

South-east

North-central

$\begin{array}{lll}1.3(0.8-2.4) & 0.8(0.4-1.3) & 1.0(0.7-1.5) \\ 0.7(0.3-1.7) & 1.2(0.6-2.2) & 0.9(0.6-1.6) \\ 0.9(0.3-3.1) & 0.1(0.0-1.0) & 0.5(0.2-1.3)\end{array}$

Family History of Prostate

Cancer

Yes

1.0

$1.3(0.8-2.0)$

1.0

$0.7(0.4-1.0)$

1.0

$0.9(0.8-1.3)$

1.0

$0.8(0.4-1.7)$

$1.2(0.3-4.8)$

$0.8(0.4-1.9)$

$1.2(0.5-3.0)$

1.0

$0.8(0.3-1.9)$

$1.4(0.3-5.9)$

$1.0(0.4-2.6)$

$0.7(0.2-2.2)$

1.0

$0.7(0.4-1.2)$

1.0

$1.9(1.1-3.4)$

$1.3(0.8-2.1)$

$0.7(0.4-1.1)$

$1.0(0.9-1.0)$

$1.0(0.1-1.0)$
$1.0(0.9-1.0)$

1.0

$0.8(0.4-1.4)$

$1.3(0.5-3.5)$

$0.9(0.5-1.7)$

$0.9(0.4-1.9)$

1.0

$1.2(0.8-1.8)$

$0.9(0.7-1.4)$

\section{Stage at Diagnosis}

Stage I

Stage II

Stage III

Stage IV

Unknown

Site of Metastasis

Lymph node

Rectum (bladder neck)

Bones

Spine

Multiple Metastasis

\section{Treatment Modality}

Surgery alone

Radiotherapy alone

Chemotherapy alone

Radiotherapy \& Surgery

Chemotherapy \& Radiotherapy

Chemotherapy \& Surgery
1.0

$0.9(0.3-3.5)$

$2.1(0.6-6.9)$

$1.0(0.4-2.6)$

$1.3(0.4-4.2)$

1.0

$0.8(0.3-2.4)$

$0.9(0.4-2.5)$

$1.1(0.4-2.8)$

$0.9(0.4-2.5)$

1.0

$1.3(0.7-2.2)$

$0.8(0.4-1.2)$

$0.4(0.1-1.7)$

$0.5(0.3-1.1)$

$0.4(0.2-0.7) *$
1.0

$0.7(0.1-3.9)$

$1.3(0.207 .8)$

$0.6(1.5-2.5)$

$1.0(0.2-6.2)$ 
Chemotherapy \& Surgery \&

$$
1.4(0.2-10.5)
$$

$3.9(0-\mathrm{CBC})$

$0.1(0.1-0.9)^{*}$

Radiotherapy

$\overline{\mathrm{CBC}}=$ can’t be computed; Overall $=2010$ - 2017 CI Confidence Interval

While controlling for other variables patients who were widowers in the 2015 - 2017 cohort experienced being disease-free earlier $(\mathrm{aHR}=5.7 ; 95 \% \mathrm{CI}: 1.4-23.1)$ compared to patients who were currently married. Meanwhile, patients with no family history of $\mathrm{CaP}$ in the same cohort experienced a $40 \%$ delay in risk of being disease-free (aHR $=0.6 ; 95 \% \mathrm{CI}: 0.3-1.0)$ compared to patients with a positive family history of $\mathrm{CaP}$. Patients with a poorly differentiated Gleason score in the $2010-2014$ cohort were disease-free later (aHR $=0.5 ; 95 \% \mathrm{CI}$ : $0.3-0.9)$ compared to patients with a well-differentiated Gleason score. Patients treated using a combination of chemotherapy and surgery had a $80 \%$ delay in risk of being disease-free (aHR $=0.2$; 95\%CI: 0.1 $-0.4)$ (table 5).

Table 5: Adjusted determinants of prostate cancer survivorship using Cox proportional hazard regression model

\begin{tabular}{l|c|c|c}
\hline Characteristic & $\mathbf{2 0 1 0}-\mathbf{2 0 1 4}$ & $\mathbf{2 0 1 5}-\mathbf{2 0 1 7}$ & Overall \\
\hline Age & aHR(95\% CI $)$ & aHR(95\% CI $)$ & aHR(95\% CI) \\
$50-59$ & 1.0 & 1.0 & 1.0 \\
$60-69$ & $0.7(0.3-1.9)$ & $1.2(0.5-2.9)$ & $1.2(0.7-2.3)$ \\
$70-79$ & $0.8(0.4-2.1)$ & $0.9(0.4-1.7)$ & $1.1(0.7-1.9)$ \\
$79-$ above & $0.7(0.2-1.8)$ & $0.7(0.3-1.7)$ & $1.2(0.7-2.2)$
\end{tabular}

\section{Marital Status}

Currently married

Widower

$1.0 \quad 1.0 \quad 1.0$

$4.4(1.1-16.5)^{*} \quad 5.7(1.4-23.1)^{*} \quad 3.7(1.4-8.1)^{*}$

\section{Occupation}

Civil Servant

Farming

Factory Worker

Unemployed

1.0

1.0

1.0

Others

$\begin{array}{cc}1.3(0.3-7.1) & 1.1(0.3-3.6) \\ 0.4(0.1-1.2) & 0.3(0.1-1.4) \\ 4.1(0.8-21.2) & 1.0(0.1-9.2) \\ 1.4(0.8-2.5) & 0.8(0.5-1.2)\end{array}$

$1.1(0.5-2.7)$

$0.5(0.2-1.1)$

$1.2(0.3-3.9)$

$0.9(0.7-1.4)$

\section{Religion}

Christian

1.0

Islam

$1.4(0.8-2.3)$

Others

$2.9(0.5-16.9)$

1.0

1.0

$1.1(0.7-1.9)$

$1.2(0.8-1.6)$

$\mathrm{CBC}$

$1.2(0.3-5.2)$ 


\section{Geographical Location}

South-west

South-south

South-east

North-central

Family History of Prostate

\section{Cancer}

Yes

No

Method of diagnosis

PSA test

DRE test

Transrectal Ultrasound

Biopsy

DRE \& Biopsy

Gleason group

Well differentiated

Moderately differentiated

Poorly differentiated

PSA level

\section{Stage at Diagnosis}

Stage I

Stage II

Stage III

Stage IV

Unknown

Site of Metastasis

Lymph node

Rectum (bladder neck)

Bones

Spine

Multiple Metastasis

Treatment Modality

Surgery

Radiotherapy

Chemotherapy

Radiotherapy \& Surgery
1.0

$1.7(0.9-3.4)$

$0.7(0.2-1.9)$

$1.5(0.3-7.7)$

1.0

$1.3(0.7-2.3)$

1.0

$0.6(0.3-1.0) *$

1.0

$0.9(0.7-1.4)$
1.0

$1.1(0.4-3.1)$

$2.2(0.4-11.3)$

$1.6(0.5-4.9)$

$1.8(0.5-6.2)$

1.0

$0.6(0.3-1.2)$

$0.5(0.3-0.9)^{*}$

$1.0(0.9-1.0)$
1.0

$1.1(0.6-1.9)$

$0.4(0.1-1.3)$

1.0

$0.6(0.3-1.2)$

$0.1(0.0-1.2)$
$1.0(0.7-1.6)$ 
Chemotherapy \&

Radiotherapy

Chemotherapy \& Surgery

Chemotherapy \& Surgery \&

Radiotherapy

NB: $C B C=$ can't be computed; Overall $=2010-2017$

\section{Parametric survival analysis (Weibull distribution)}

Result from the bivariate Weibull regression model showed that patients who were widowers in the 2015 - 2017 cohort experienced a significant increase of $70 \%$ in time to being disease-free (TR $=0.3 ; 95 \%$ CI: $0.1-0.9)$ compared to patients currently married (Table 6).

Patients with a moderately differentiated Gleason score experienced a significant increase of $50 \%$ in time to being disease-free ( $\mathrm{TR}=0.5$; 95\%CI: $0.3-0.9$ ) for those in the $2015-2017$ cohort.

Being treated by chemotherapy and radiotherapy significantly decreased time to being disease-free by $60 \%$ (TR $=1.6 ; 95 \% \mathrm{CI}: 1.0-2.6$ ) for the overall group, compared to patients who underwent surgery. In like manner patients who received chemotherapy and surgery had a significant delay in time to being disease-free (TR $=2.4 ; 95 \%$ CI: $1.4-3.9)$ for the $2010-2014$ cohort, also for the overall group, time to being disease-free was delayed ( $\mathrm{TR}=1.9 ; 95 \% \mathrm{CI}: 1.4-2.9)$ compared to patients who underwent surgery. Patients who received a combination of chemotherapy, surgery and radiotherapy also experienced a delay in time to being disease-free (TR $=7.0 ; 95 \% \mathrm{CI}: 1.0-$ 49.2) compared to patients who underwent surgery (Table 6).

Table 6: Unadjusted determinants of prostate cancer survivorship using Weibull Parametric Regression

\begin{tabular}{l|c|c|c}
\hline Characteristic & $\mathbf{2 0 1 0}-\mathbf{2 0 1 4}$ & $\mathbf{2 0 1 5}-\mathbf{2 0 1 7}$ & Overall \\
\hline Age & TR(95\% CI $)$ & TR(95\% CI) & TR(95\% CI) \\
$50-59$ & 1.0 & 1.0 & 1.0 \\
$60-69$ & $0.9(0.4-1.9)$ & $1.1(0.5-2.3)$ & $1.0(0.6-1.7)$ \\
$70-79$ & $0.9(0.5-1.8)$ & $1.0(0.5-1.9)$ & $0.9(0.6-1.6)$ \\
$79-$ above & $0.7(0.3-1.5)$ & $1.1(0.6-2.2)$ & $0.9(0.6-1.5)$
\end{tabular}

Marital Status

Currently married

Widower

1.0

$0.5(0.2-1.6)$
1.0 $0.3(0.1-0.9)^{*}$ 1.0 
Occupation

Civil Servant

Farming

Factory Worker

Unemployed

Others

$\begin{array}{ccc}1.0 & 1.0 & 1.0 \\ 0.8(0.2-2.9) & 0.8(0.3-2.0) & 0.8(0.3-1.7) \\ 1.6(0.7-3.7) & 2.8(0.7-11.5) & 1.9(0.9-3.9) \\ 0.9(0.2-3.3) & 1.7(0.2-11.9) & 1.1(0.4-3.5) \\ 0.9(0.7-1.4) & 1.3(0.9-1.8) & 1.1(0.9-1.5)\end{array}$

Religion

Christian

Islam

1.0

$0.6(0.4-0.9)$

1.0

$1.1(0.7-1.7)$

1.0

Others

$0.6(0.1-2.1)$

CBC

$0.9(0.6-1.1)$

$1.1(0.3-4.2)$

Geographical Location

South-west

South-south

South-east

North-central

1.0

1.0

1.0

$0.7(0.4-1.3)$

$1.3(0.7-2.2)$

$0.9(0.7-1.5)$

$1.4(0.6-3.5)$

$0.9(0.5-1.5)$

$1.0(0.6-1.7)$

$1.0(0.3-3.2)$

$2.9(0.7-11.6)$

$1.8(0.8-4.3)$

Family History of Prostate

\section{Cancer}

Yes

$\begin{array}{ccc}1.0 & 1.0 & 1.0 \\ 0.8(0.5-1.2) & 1.4(0.9-2.2) & 1.1(0.8-1.4)\end{array}$

\section{Method of diagnosis}

PSA test

1.0

$1.3(0.6-2.7)$

1.0

1.0

DRE test

Transrectal Ultrasound

$0.8(0.2-2.9)$

$1.3(0.5-3.2)$

$1.3(0.7-2.3)$

$1.2(0.5-2.6)$

$0.7(0.2-2.9)$

$0.8(0.3-1.9)$

$0.8(0.3-2.0)$

$0.9(0.4-2.5)$

$1.1(0.6-1.9)$

DRE \& Biopsy

$1.5(0.5-4.5)$

$1.1(0.5-2.1)$

\section{Gleason group}

Well differentiated

Moderately differentiated

Poorly differentiated

1.0

$1.4(0.8-2.5)$

$1.4(0.8-2.2)$

$0.9(0.9-1.0)$

1.0

$1.1(0.3-3.9)$

$0.5(0.2-1.6)$

$0.9(0.4-2.3)$

$0.8(0.3-2.5)$
1.0

$0.5(0.3-0.9)^{*}$

$0.8(0.5-1.2)$

$1.0(0.9-1.0)$

1.0

$1.4(0.3-7.5)$

$0.8(0.1-4.4)$

$1.6(0.4-6.2)$

$0.8(0.2-5.2)$
1.0

$0.8(0.6-1.3)$

$1.0(0.7-1.4)$

$0.9(0.9-1.0)$

Stage IV

Unknown

Site of Metastasis 
Lymph node

Rectum (bladder neck)

Bones

Spine

Multiple Metastasis
1.0

$1.1(0.4-3.0)$

$1.0(0.4-2.5)$

$0.9(0.4-2.2)$

$1.0(0.4-2.6)$
1.0

$0.9(0.3-2.1)$

$0.9(0.4-2.1)$

$0.9(0.4-2.1)$

$0.8(0.3-2.1)$
1.0

$0.9(0.5-1.9)$

$0.9(0.5-1.7)$

$0.9(0.5-1.6)$

$0.9(0.5-1.8)$

Treatment Modality

Surgery

1.0

$0.8(0.5-1.4)$

$1.4(0.9-2.5)$

$2.4(0.6-8.9)$

$1.7(0.9-3.6)$

$2.4(1.4-3.9)^{*}$

$0.8(0.1-4.3)$
1.0

$1.5(0.8-2.7)$

$0.9(0.6-1.6)$

$2.3(0.6-9.3)$

$1.5(0.8-2.8)$

$1.6(0.9-2.7)$

$0.4(0-\mathrm{CBC})$
1.0

$1.1(0.7-1.6)$

$1.2(0.8-1.7)$

$2.3(0.9-6.2)$

$1.6(1.0-2.6)^{*}$

$1.9(1.4-2.9)^{*}$

$7.0(1.0-49.2)^{*}$

Radiotherapy

$\mathrm{CBC}=$ can't be computed; Overall $=2010-2017 ;$ TR Time Ratio

Result from the multivariate analysis showed that patients who were widowers experienced a $80 \%$ increase in time to being disease-free in the $2015-2017$ cohort (aTR $=0.2$; 95\% CI: $0.1-0.7$ ) and also a 70\% increase in the $2010-2014$ cohort and overall group (aTR $=0.3 ; 95 \%$ CI: $0.1-$ $0.8)$ and (aTR $=0.3 ; 95 \%$ CI: $0.1-0.7)$ compared to patients who are currently married (Table 7).

Time to being disease-free significantly decreased by $70 \%$ for patients with a poorly differentiated Gleason score in the $2010-2014$ cohort (aTR $=1.7 ; 95 \%$ CI: $1.0-2.7$ ) compared to patients with a well-differentiated gleason score. Patients diagnosed at stage III also in the same cohort experienced a significant increase of $60 \%$ in time to being disease-free (aTR $=0.4 ; 95 \%$ CI: 0.1 1.0) compared to patients diagnosed at stage I. Patients in the $2010-2014$ cohort who were treated using a combination of chemotherapy and surgery experienced a $220 \%$ delay in time to being disease-free (aTR $=3.2 ; 95 \%$ CI: $1.9-5.3$ ). Those treated using a combination chemotherapy and radiotherapy in the overall group also had a significant delay of $80 \%$ in time to being disease-free $(\mathrm{aTR}=1.8 ; 95 \%$ CI: $1.1-3.1)$. Likewise, time to being disease-free significantly decreased by $90 \%$ for patients treated using a combination of chemotherapy and surgery in the overall group respectively (aTR $=1.9 ; 95 \%$ CI: $1.4-2.8$ ) compared to patients who underwent surgery alone (Table 4.7). 
Table 7: Adjusted determinants of prostate cancer survivorship using Weibull Parametric Regression model

\begin{tabular}{|c|c|c|c|}
\hline Characteristic & $2010-2014$ & $2015-2017$ & Overall \\
\hline & $\operatorname{aTR}(95 \% \mathrm{CI})$ & $\operatorname{aTR}(95 \% \mathrm{CI})$ & $\operatorname{aTR}(95 \% \mathrm{CI})$ \\
\hline \multicolumn{4}{|l|}{ Age } \\
\hline $50-59$ & 1.0 & 1.0 & 1.0 \\
\hline $60-69$ & $1.2(0.6-2.6)$ & $0.9(0.4-2.0)$ & $0.8(0.5-1.5)$ \\
\hline $70-79$ & $1.0(0.5-2.1)$ & $1.1(0.6-2.2)$ & $0.9(0.5-1.4)$ \\
\hline 79 - above & $1.3(0.6-3.1)$ & $1.3(0.7-2.8)$ & $0.8(0.5-1.4)$ \\
\hline
\end{tabular}

\section{Marital Status}

Currently married

Widower

$$
\begin{array}{ccc}
1.0 & 1.0 & 1.0 \\
0.3(0.1-0.8)^{*} & 0.2(0.1-0.7)^{*} & 0.3(0.1-0.7)^{*}
\end{array}
$$

Occupation

Civil Servant

Farming

Factory Worker

Unemployed

Others

$\begin{array}{ccc}1.0 & 1.0 & 1.0 \\ 0.8(0.2-2.9) & 1.0(0.3-2.9) & 0.9(0.4-2.0) \\ 2.1(0.9-4.8) & 2.7(0.7-10.4) & 1.9(0.9-3.8) \\ 0.4(0.1-1.4) & 0.9(0.1-7.1) & 0.9(0.3-2.9) \\ 0.8(0.5-1.2) & 1.2(0.8-1.9) & 1.0(0.8-1.4)\end{array}$

\section{Religion}

Christian

Islam

1.0

$0.8(0.5-1.2)$

Others
1.0

$0.9(0.5-1.4)$

$\mathrm{CBC}$
1.0

$0.9(0.6-1.1)$

$0.9(0.2-3.4)$

\section{Geographical Location}

South-west

South-south

South-east

North-central
1.0

$0.7(0.4-1.1)$

$1.3(0.6-2.9)$

$0.7(0.2-2.7)$
1.0

$1.4(0.8-2.6)$

$1.0(0.4-2.3)$

$3.3(0.7-14.9)$
1.0

$0.9(0.7-1.5)$

$0.9(0.5-1.6)$

$2.2(0.8-5.8)$

\section{Family History of Prostate}

\section{Cancer}

Yes

$\begin{array}{ccc}1.0 & 1.0 & 1.0 \\ 0.8(0.5-1.3) & 1.5(0.9-2.4) & 1.0(0.7-1.4)\end{array}$

\section{Method of diagnosis}

PSA test

1.0

DRE test

$0.9(0.4-2.2)$
$0.5(0.1-1.9)$
$0.7(0.3-1.6)$
$0.6(0.2-1.7)$

1.0

1.0

Transrectal Ultrasound

$$
1.4(0.5-3.9)
$$

$1.4(0.8-2.6)$

$0.9(0.2-5.3))$

$0.8(0.3-2.3)$

Biopsy

DRE \& Biopsy

$0.9(0.3-2.8)$

$1.1(0.5-2.1)$

$1.9(0.6-7.2)$

$1.3(0.6-2.8)$

\section{Gleason group}

Well differentiated 
Moderately differentiated

Poorly differentiated

PSA level

Stage at Diagnosis

Stage I

Stage II

Stage III

Stage IV

Unknown

Site of Metastasis

Lymph node

Rectum (bladder neck)

Bones

Spine

Multiple Metastasis

Treatment Modality

Surgery

Radiotherapy

Chemotherapy

Radiotherapy \& Surgery

Chemotherapy \& Radiotherapy

Chemotherapy \& Surgery

Chemotherapy \& Surgery \&

Radiotherapy

$$
\begin{array}{ccc}
1.4(0.8-2.5) & 0.6(0.3-1.1) & 0.8(0.5-1.2) \\
1.7(1.0-2.7)^{*} & 0.7(0.4-1.2) & 1.0(0.7-1.4) \\
& & \\
0.9(0.9-1.0) & 1.0(0.9-1.0) & 0.9(0.9-1.0)
\end{array}
$$

\section{$\begin{array}{lll}1.0 & 1.0 & 1.0\end{array}$}

$\begin{array}{ccc}0.5(0.1-1.9) & 0.9(0.1-5.6) & 0.7(0.3-1.9) \\ 0.4(0.1-1.0)^{*} & 0.5(0.1-3.7) & 0.4(0.1-1.1) \\ 0.5(0.2-1.3) & 1.2(0.2-5.9) & 0.8(0.4-1.8) \\ 0.5(0.1-1.5) & 1.3(0.5-3.6) & 0.7(0.2-1.9)\end{array}$

1.0

$0.9(0.3-2.6)$

$0.9(0.4-2.5)$

$0.7(0.3-1.9)$

$1.1(0.4-2.9)$
1.0

$1.1(0.4-3.0)$

$1.5(0.6-4.1)$

$1.3(0.5-3.5)$

$1.3(0.5-3.6)$
1.0

$0.9(0.4-1.7)$

$0.9(0.5-1.7)$

$0.9(0.5-1.6)$

$0.9(0.5-1.8)$

\begin{tabular}{lccc} 
_cons & $6.3(1.5-27.4)$ & $1.7(0.2-17.9)$ & $4.2(1.2-14.4)$ \\
/In_p & $0.2(0.1-0.4)$ & $0.1(-0.1-0.2)$ & $0.1(-0.0-0.2)$ \\
$\mathrm{P}$ & $1.2(1.1-1.5)$ & $1.1(0.9-1.3)$ & $1.1(0.9-1.2)$ \\
$1 / \mathrm{p}$ & $0.8(0.7-0.9)$ & $0.9(0.8-1.1)$ & $0.9(0.8-1.0)$ \\
\hline $\mathrm{CBC}=$ can't be computed; Overall = $2010-2017 ;$ aTR $=$ adjusted Time Ratio &
\end{tabular}

\section{Bayesian survival analysis (Weibull distribution)}

Results from the bivariate Bayesian parametric (Weibull) regression model showed that men who were factory workers experienced a $20 \%$ delay in time to being disease-free (TR $=2.2 ; 95 \%$ Credible Interval (CrI: $1.1-4.3)$ ) compared to individuals who are civil servants (Table 8). 
Patients with a moderately differentiated Gleason score in the 2015 - 2017 cohort experienced a significant increase in time to being disease-free by $60 \%$ (TR $=0.6 ; 95 \% \mathrm{CrI}: 0.3-0.9$ ) compared to patients with a well-differentiated Gleason score (table 8).

Patients treated using a combination of radiotherapy and surgery in the overall group experienced a significant delay of $90 \%$ in time to being disease-free (TR $=2.9 ; 95 \% \mathrm{CrI}: 1.1-7.5)$ compared to patients who underwent surgery alone. Meanwhile, treatment via chemotherapy and surgery for both the $2010-2014$ cohort significantly delayed time to being disease-free $(\mathrm{TR}=2.0 ; 95 \% \mathrm{CrI}$ : 1.4 - 4.2); compared to patients who underwent surgery alone. Patients who received all three combinations of chemotherapy, surgery and radiotherapy experienced delay in time to being disease-free for the 2015 - 2017 cohort and the overall group, compared to patients who underwent surgery alone (Table 8).

Table 8: Unadjusted determinants of prostate cancer survivorship using Bayesian Parametric (Weibull distribution) Regression model

\begin{tabular}{|c|c|c|c|}
\hline Characteristic & $2010-2014$ & 2015 - 2017 & Overall \\
\hline & TR(95\% CrI) & TR(95\% CrI) & TR(95\% CrI) \\
\hline \multicolumn{4}{|l|}{ Age } \\
\hline $50-59$ & 1.0 & 1.0 & 1.0 \\
\hline $60-69$ & $0.9(0.4-1.7)$ & $1.1(0.5-2.2)$ & $0.9(0.5-1.5)$ \\
\hline $70-79$ & $0.9(0.4-1.7)$ & $1.0(0.5-1.9)$ & $0.9(0.6-1.4)$ \\
\hline 79 - above & $0.7(0.3-1.4)$ & $1.1(0.5-2.1)$ & $0.9(0.5-1.4)$ \\
\hline \multicolumn{4}{|l|}{ Marital Status } \\
\hline Married & 1.0 & 1.0 & 1.0 \\
\hline Widower & $0.8(0.2-2.5)$ & $0.5(0.1-1.5)$ & $0.5(0.2-1.1)$ \\
\hline \multicolumn{4}{|l|}{ Occupation } \\
\hline Civil Servant & 1.0 & 1.0 & 1.0 \\
\hline Farming & $1.5(0.3-6.9)$ & $1.1(0.4-3.0)$ & $0.9(0.4-2.1)$ \\
\hline Factory Worker & $1.9(0.8-4.8)$ & $6.7(0.9-28.6)$ & $2.2(1.1-4.3)^{*}$ \\
\hline Unemployed & $1.7(0.3-6.9)$ & $13.9(0.5-103.1)$ & $1.7(0.4-5.8)$ \\
\hline Others & $0.9(0.7-1.5)$ & $1.3(0.8-1.8)$ & $1.1(0.8-1.5)$ \\
\hline \multicolumn{4}{|l|}{ Religion } \\
\hline Christian & 1.0 & 1.0 & 1.0 \\
\hline Islam & $0.7(0.4-1.0)$ & $1.4(0.7-1.8)$ & $0.9(0.6-1.2)$ \\
\hline Others & $1.2(0.1-4.9)$ & $1.2(4.6-3.7)^{*}$ & $2.0(0.4-8.9)$ \\
\hline
\end{tabular}

\section{Geographical Location}


South-west

South-south

South-east

North-central
1.0

$0.8(0.5-1.5)$

$1.8(0.7-4.6)$

$1.6(0.4-4.9)$
1.0

$1.3(0.7-2.4)$

$0.9(0.5-1.7)$

$5.3(1.0-21.2)$
1.0

$1.0(0.7-1.5)$

$1.1(0.7-1.8)$

$2.2(0.9-5.6)$

\section{Family History of Prostate}

\section{Cancer}

Yes

1.0

No

$0.8(0.5-1.2)$

1.0

$1.4(0.8-2.1)$

1.0

$1.0(0.7-1.4)$

\section{Method of diagnosis}

PSA test

1.0

DRE test

$1.3(0.5-2.4)$

1.0

Transrectal Ultrasound

$1.2(0.3-4.2)$

$1.2(0.5-2.4)$

$0.9(0.3-1.9)$

$1.3(0.4-2.8)$

$1.1(0.2-4.0)$

$0.9(0.3-2.2)$

$1.7(0.4-4.8)$

1.0

$1.3(0.6-2.2)$

$0.9(0.3-2.2)$

$1.1(0.5-1.9)$

$1.1(0.5-2.1)$

DRE \& Biopsy

1.0

$1.5(0.8-2.6)$

$1.4(0.9-2.2)$

$1.0(0.9-1.0)$

1.0

$0.6(0.1-1.6)$

$0.9(0.3-2.1)$

$0.9(0.2-2.6)$

$\mathrm{CBC}$
1.0

$0.6(0.3-0.9)^{*}$

$0.8(0.5-1.2)$

$1.0(0.9-1.0)$

$2.0(0.1-5.6)$

$1.6(0.1-4.7)$

$1.4(0.1-6.1)$

$\mathrm{CBC}$

1.0

$1.2(0.3-2.9)$

$1.0(0.3-2.3)$

$0.9(0.3-2.0)$

$1.1(0.3-2.4)$
1.0

$0.9(0.3-1.9)$

$0.9(0.3-1.9)$

$0.9(0.3-1.9)$

$0.9(0.3-1.9)$
1.0

$0.9(0.7-1.3)$

$1.0(0.7-1.4)$

$1.0(0.9-1.0)$

Spine

Multiple Metastasis

Treatment Modality

Surgery

Radiotherapy

Chemotherapy

Radiotherapy \& Surgery

Chemotherapy \& Radiotherapy

Chemotherapy \& Surgery
1.0

$0.8(0.5-1.3)$

$1.5(0.9-2.5)$

$3.5(0.8-11.1)$

$2.0(0.9-3.8)$

$2.0(1.4-4.2)^{*}$
1.0

$1.6(0.8-3.2)$

$0.9(0.5-1.7)$

$5.7(0.8-26.9)$

$1.8(0.8-3.8)$

$1.8(0.9-3.2)$

\section{0}

$1.3(0.4-3.1)$

$0.6(0.2-1.3)$

$1.1(0.5-2.1)$

$0.9(0.3-2.3)$

\section{0}

$1.0(0.5-1.8)$

$0.9(0.4-1.7)$

$0.9(0.4-1.6)$

$0.9(0.4-1.7)$ 
Chemotherapy \& Surgery \& $\quad 4.5(0.2-20.4) \quad 4.2(18.3-1.6)^{*} \quad 40.4(1.7-291.6)^{*}$ Radiotherapy

NB: $\mathrm{CBC}=$ can’t be computed; Overall $=2010-2017$; CrI = Credible Interval

While adjusting for other variables, patients age $60-69$ years and 79 years and above in the 2010 - 2014 cohort experienced a significant delay of 30\% and 40\% respectively in time to being disease-free $(\mathrm{aTR}=1.3 ; 95 \% \mathrm{CrI}: 1.1-1.5) ;(\mathrm{aTR}=1.4 ; 95 \% \mathrm{CrI}: 1.1-1.8)$. Widowers experienced a significant increase of $80 \%, 70 \%$ and $80 \%$ in time to being disease-free for the 2010 - 2014, and 2015 - 2017 cohorts and overall group (aTR =0.3; 95\%CrI: $0.2-0.3$ ), (aTR =0.2; 95\% CrI: $0.1-0.3$ ), and (aTR $=0.3 ; 95 \% \mathrm{CrI}: 0.2-0.4)$ compared to patients who were currently married. Time to being disease-free more than delayed for patients who were factory workers for the $2015-2017$ cohort (aTR $=2.9 ; 95 \%$ CrI: $1.7-4.7$ ) meanwhile patients who were unemployed experienced a $40 \%$ increase in time to being disease-free for the $2010-2014$ cohort (TR $=0.4$; 95\%CrI: $0.4-0.4)$ compared to patients who were civil servants. Patients from the south-south and north-central experienced $40 \%$ and $230 \%$ delay in time to being disease-free respectively for the $2015-2017$ cohort (TR $=1.4 ; 95 \%$ CrI: $1.1-1.7)$, (TR $=3.3 ; 95 \%$ CrI: $2.1-4.9)$ compared to patients from the south-west. However, for the $2010-2014$ cohort, patients from north-central experienced a significant increase of 30\% in time to being disease-free (aTR $=0.7 ; 95 \% \mathrm{CrI}$ : 0.6 - 0.8) compared to patients from the south-west (Table 9).

Patients with no family history of $\mathrm{CaP}$ experienced a significant delay of 50\% in time to being disease-free for the 2015 - 2017 cohort, compared to patients with a positive family history of $\mathrm{CaP}$ $($ aTR $=1.5 ; 95 \%$ CrI: $1.2-2.0)$. Patients diagnosed by a combination of DRE and biopsy experienced a delay of $10 \%$ in time to being disease-free ( $\mathrm{aTR}=2.1$; $95 \% \mathrm{CrI}: 1.5-2.7)$ for the 2015 - 2017 cohort compared to patients diagnosed via PSA test. Meanwhile, for the 2010-2014 cohort, patients diagnosed via transrectal ultrasound, biopsy and a combination of DRE and Biopsy experienced 50\%, 70\% and 60\% experienced being disease-free earlier, compared to patients who were diagnosed via PSA test.

Patients with a moderately differentiated Gleason score for the 2010 - 2014 cohort experienced a $40 \%$ decrease in time to being disease-free ( $\mathrm{aTR}=1.4 ; 95 \% \mathrm{CrI}: 1.3-1.6$ ), similarly patients with a poorly differentiated Gleason score experienced $70 \%$ delay in time to being disease-free (aTR $=$ 
1.7; $95 \%$ CrI: 1.3 - 2.2) compared to patients with a well-differentiated Gleason score. Patients diagnosed at stage III for the 2015 - 2017 cohort time to being disease-free increased by 50\% (aTR $=0.5 ; 95 \%$ CrI: $0.3-0.8$ ) compared to patients diagnosed at stage I. Meanwhile patients diagnosed at stage II, III and IV experienced 50\%, 40\%, and 50\% increase in time to being disease-free compared to patients diagnosed at stage I. patients with metastasis to the bones and spine in the 2015 - 2017 cohort experienced 50\% and 30\% delay in their time to being disease-free (aTR = 1.5; 95\%CrI: $1.1-2.1)$ and (aTR $=1.3 ; 95 \% \mathrm{CrI}: 1.1-1.6)$ while patients in the $2010-2014$ cohort and overall group experienced $30 \%$ increase in time to being disease-free respectively (aTR $=0.7 ; 95 \% \mathrm{CrI}: 0.6-0.9)$ compared to patients with metastasis to the lymph node.

Patients who were treated using a combination of chemotherapy and radiotherapy experienced a $70 \%$ delay in time to being disease-free compared to patients who underwent surgery for the 2015 - 2017 cohort $($ aTR $=1.7$; 95\%CrI: 1.2 - 2.3). Patients who received radiotherapy alone experienced a $70 \%$ increase in time to being disease-free (aTR $=0.7 ; 95 \% \mathrm{CrI}: 0.7-0.8)$ for the 2010 - 2014 cohort compared to patients who underwent surgery. However, individuals who were treated using chemotherapy, radiotherapy and surgery, chemotherapy and radiotherapy, chemotherapy and surgery experienced $20 \%, 20 \%, 60 \%$ and $30 \%$ delay in time to being diseasefree for the 2010 - 2014 cohort compared to patients who underwent surgery alone (table 9).

Table 9: Adjusted determinants of prostate cancer survivorship using Bayesian Parametric (Weibull Distribution) Regression model

\begin{tabular}{lccc}
\hline Characteristic & $\mathbf{2 0 1 0}-\mathbf{2 0 1 4}$ & $\mathbf{2 0 1 5}-\mathbf{2 0 1 7}$ & Overall \\
\hline Age & aTR(95\% CrI) & aTR(95\% CrI) & aTR(95\%CrI) \\
$50-59$ & 1.0 & 1.0 & 1.0 \\
$60-69$ & $* 1.3(1.1-1.5)$ & $1.3(0.4-2.8)$ & $0.9(0.8-1.2)$ \\
$70-79$ & $1.1(0.8-1.4)$ & $1.3(0.5-2.2)$ & $0.9(0.8-1.2)$ \\
$79-$ above & $* 1.4(1.1-1.8)$ & $1.9(0.7-3.5)$ & $0.9(0.7-1.3)$ \\
& & & \\
Marital Status & & & 1.0 \\
Married & 1.0 & 1.0 & $0.3(0.2-0.4)^{*}$ \\
Widower & $0.3(0.2-0.3)^{*}$ & $0.2(0.1-0.3)^{*}$ & \\
& & & 1.0 \\
Occupation & & 1.0 & $1.1(0.5-1.8)$ \\
Civil Servant & 1.0 & $1.0(0.8-1.4)$ & $1.9(1.0-3.1)$ \\
Farming & $0.8(0.6-1.1)$ & $2.9(1.7-4.7)^{*}$ &
\end{tabular}


Unemployed

Others

\section{Religion}

Christian

Islam

Others

Geographical Location

South-west

South-south

South-east

North-central

\section{Family History of Prostate}

\section{Cancer}

Yes

No

\section{Method of diagnosis}

PSA test

DRE test

Transrectal Ultrasound

Biopsy

DRE \& Biopsy

\section{Gleason group}

Well differentiated

Moderately differentiated

Poorly differentiated

PSA level

\section{Stage at Diagnosis}

Stage I

Stage II

Stage III

Stage IV

Unknown

Site of Metastasis

Lymph node

Rectum (bladder neck)

Bones

Spine

Multiple Metastasis

$$
0.4(0.4-0.4) * \quad 1.0(0.7-1.4)
$$

$1.2(0.9-1.5)$

$0.6(0.4-1.6)$

$0.8(0.7-0.8) *$

1.0

$0.8(0.7-0.9) *$

$0.4(0.3-0.6)$

1.0

$0.8(0.7-1.2)$

CBC

1.0

$0.9(0.7-1.3)$

$1.2(0.8-1.7)$
1.0

$0.7(0.5-1.1)$

$1.3(0.9-1.7)$

$0.7(0.6-0.8) *$

1.0

$0.8(0.7-0.9) *$

1.0

$0.9(0.9-1.0)$

$0.5(0.4-0.7)^{*}$

$0.7(0.6-0.8)^{*}$

$0.6(0.5-0.8)^{*}$

1.0

$1.4(1.3-1.6)^{*}$

$1.7(1.3-2.2)^{*}$

$0.9(0.9-1.0)$

1.0

$0.5(0.4-0.6)^{*}$

$0.4(0.3-0.4)^{*}$

$0.5(0.4-0.7)^{*}$

$0.5(0.4-0.6) *$

1.0

$0.9(0.6-1.4)$

$0.9(0.7-1.2)$

$0.7(0.6-0.9)^{*}$

$1.1(0.9-1.3)$
1.0

$1.4(1.1-1.7)^{*}$

$1.0(0.9-1.3)$

$3.3(2.1-4.9)^{*}$

1.0

$1.5(1.2-2.0)^{*}$

1.0

$1.1(0.7-1.4)$
1.0

$0.9(0.7-1.3)$

$1.0(0.7-1.6)$

$2.6(1.8-3.5)$
1.0

$1.4(0.9-1.9)$

$0.9(0.6-1.4)$

$0.9(0.7-1.2)$

2.1(1.5-2.7)*

1.0

$0.6(0.4-0.8) *$

$0.8(0.6-0.9)^{*}$

$1.0(0.9-1.0)$

1.0

$0.9(0.7-1.2)$

$0.5(0.3-0.8)^{*}$

$1.2(1.0-1.4)$

$0.9(0.6-1.4)$

1.0

$1.1(0.8-1.4)$

$1.5(1.1-2.1)^{*}$

$1.3(1.1-1.6)^{*}$

$1.3(0.9-1.9)$
1.0

$1.4(1.0-1.9)$

$0.8(0.4-1.4)$

$1.1(0.8-1.4)$

$1.4(0.9-1.9)$ 


\section{Treatment Modality}

Surgery

Radiotherapy

1.0

$0.7(0.7-0.8)^{*}$

1.0

1.0

Chemotherapy

$1.2(1.5-2.1)^{*}$

$1.0(0.8-1.4)$

$0.9(0.6-1.3)$

$3.2(2.3-4.4)^{*}$

$0.9(0.8-1.2)$

$1.1(0.7-1.7)$

Radiotherapy \& Surgery

$1.6(1.3-1.9)^{*}$

$1.4(0.9-2.0)$

$2.4(1.3-4.5)^{*}$

Chemotherapy \& Radiotherapy

$3.3(2.6-4.4)^{*}$

$1.7(1.2-2.3)^{*}$

$2.1(1.3-2.9)^{*}$

Chemotherapy \& Surgery

$0.5(0.4-0.8)^{*}$

$1.2(0.9-1.6)$

$1.9(1.4-2.7)^{*}$

Chemotherapy \& Surgery \&

Radiotherapy

CBC

$10.4(4.7-16.7)^{*}$

baseline time

$6.2(5.2-7.3)$

$1.8(1.3-2.2)$

$5.5(2.3-11.9)$

In_p

$0.2(0.0-0.3) \quad 0.0(-0.1-0.2)$

$-0.0(-0.1-0.1)$

NB: CBC = can't be computed; Overall $=2010-2017 ; \mathrm{CrI}=$ Credible Interval

\section{Model adequacy checks}

The fitness of the models considered in the multivariate analysis was determined to use $-2 L L, A I C$, BIC and DIC. In addition, convergence for the Bayesian model was determined by acceptance rate and graphical diagnostics using trace, histogram, autocorrelation and kernel density plots.

Model check for the Cox proportional hazard regression it was observed that the model for the 2010 - 2014 cohort of patients was the best fitting model with consistently lesser values of $-2 L L$, AIC and DIC. For the parametric Accelerated (Weibull distribution) Failure Time regression, the model for the 2010 - 2014 cohort of patients was observed as the best fitting model. Adequacy check for the Bayesian AFT regression showed that the best fitting model for the Bayesian analysis is the model of the $2010-2014$ cohort of patients (Table 10).

While checking for the significant parameters in the Bayesian AFT regression, it was observed that most parameters in the 2010 - 2014 cohort model were significant. Observing their MCMC convergence, the result showed that stage I and II levels under the parameter stage at diagnosis converged more as can be shown in the trace plot. A trace plot, plots the values of the simulated parameters against the iteration number and connect consecutive values with a line. For a convergent parameter, the drawn line looks almost vertical and dense, while sparseness and trends suggest convergence problem. The histogram is plotted in relation to the selected prior distribution, in this study a normally distributed prior was selected as such the histogram plot should be normally distributed as deviation from normality indicates convergence issues. The kernel density 
plot or smoothed histogram shows three density plots first, second and overall of an MCMC sample, a closely matched plots indicates convergence, while large discrepancies between the halfs' suggests convergence problem (figure 4). Convergence was best among patients who received chemotherapy for the 2010 - 2014 cohort (figure 5).

Table 10: Model Adequacy Check

\begin{tabular}{|c|c|c|c|}
\hline Criteria & $2010-2014$ & $2015-2017$ & Overall \\
\hline & \multicolumn{3}{|c|}{ Cox Proportional Hazard model (Adjusted) } \\
\hline$-2 \mathbf{L L}$ & 863.8 & 927.9 & 2147.2 \\
\hline $\mathbf{A I C}$ & 931.8 & 991.9 & 2215.1 \\
\hline \multirow[t]{2}{*}{ BIC } & 1037.2 & 1095.8 & 2347.1 \\
\hline & \multicolumn{3}{|c|}{ Parametric Accelerated Failure Time model (Adjusted) } \\
\hline$-2 \mathbf{L L}$ & 376.3 & 435.4 & 858.1 \\
\hline AIC & 448.3 & 507.4 & 930.1 \\
\hline \multirow[t]{2}{*}{ BIC } & 559.9 & 624.3 & 1069.4 \\
\hline & Bayesian Para & elerated Fail & del (Adjusted) \\
\hline$-2 \mathrm{LL}$ & 959.3 & 900.8 & 1281.2 \\
\hline DIC & 394.7 & 456.0 & 908.7 \\
\hline BIC & 932.7 & 928.2 & 1311.8 \\
\hline $\mathbf{A R}$ & 0.4 & 0.3 & 0.3 \\
\hline Efficiency & 0.2 & 0.1 & 0.1 \\
\hline
\end{tabular}

NB: $A R=$ Acceptance Rate; Overall = $2010-2017$ 
Figure 4: MCMC Convergence diagnostics for selected characteristics (2010 - 2014) cohort

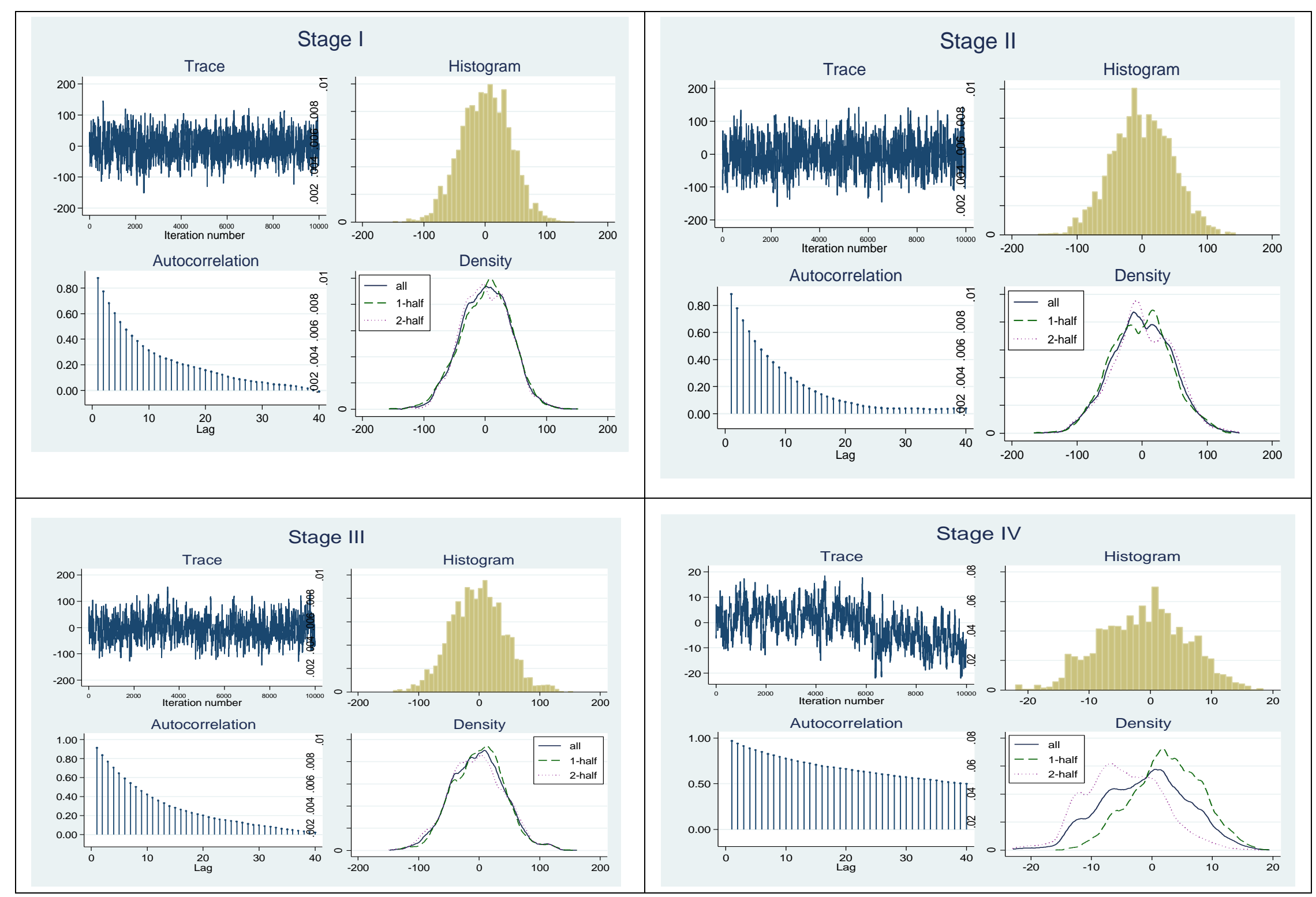


Figure 5: MCMC Convergence diagnostics for selected characteristics (2010 - 2014) cohort

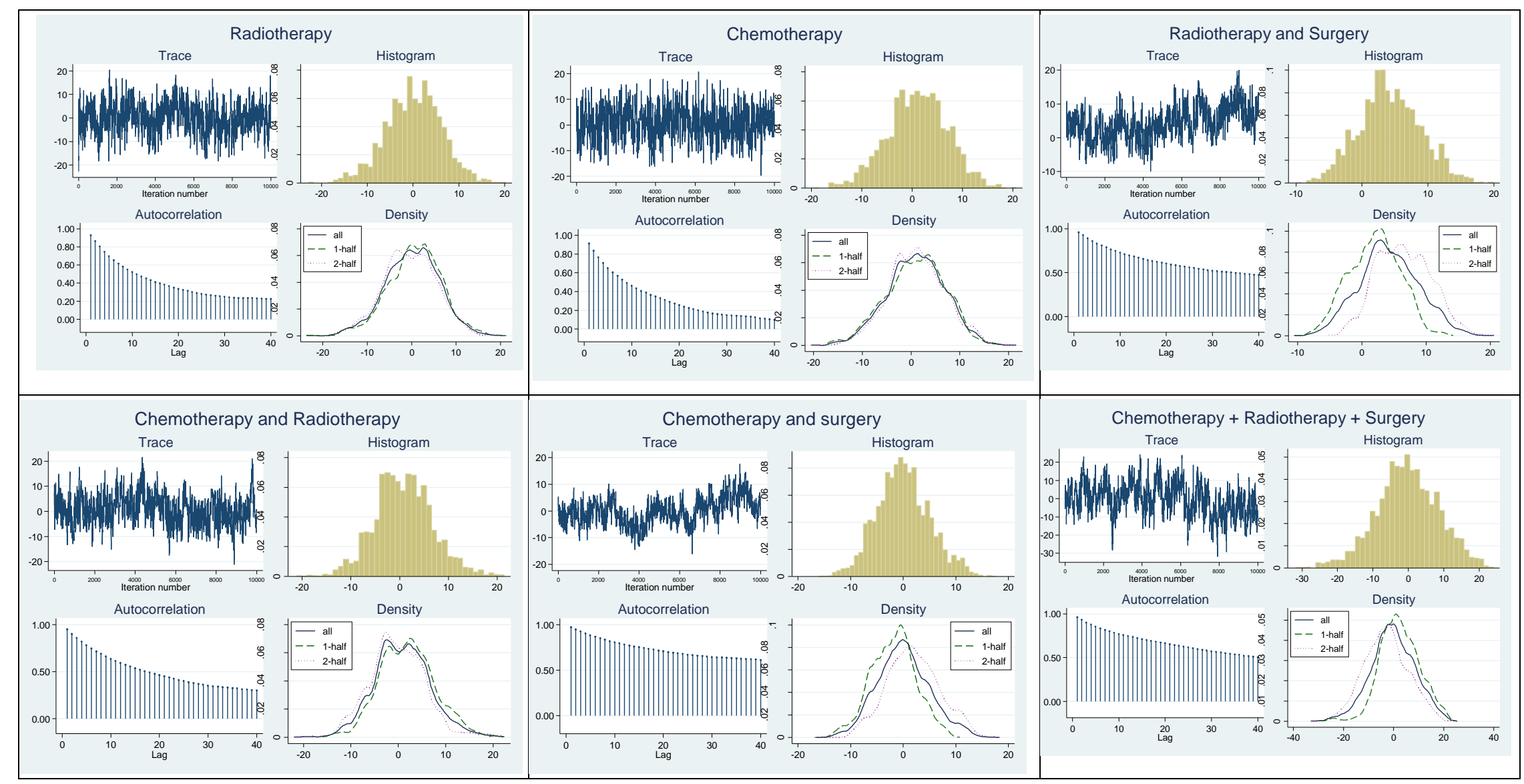




\section{DISCUSSION}

This study accessed the rate of survivorship and determinants of survivorship among CaP patients. The incidence of $\mathrm{CaP}$ was highest among patients older than seventy-two years. This is consistent with studies of (Ifere, Abebe, \& Ananaba, 2012). CaP is known to be a disease of the aged with very few incidences observed among those below forty years, and very recurring as men progress in age. Advanced age is associated with an aggressive form of this disease (Ji et al., 2017) and a predictor for decreased survival rate as also shown in the Bayesian analysis of this study.

It was observed that patients currently married had longer survival after treatment compared to widowers, this could be due to support received from spouse and family members. Particularly in an African setting where cancer is seen as a death sentence, support from family members and a feeling of not being left alone could encourage quick recovery (Odedina et al., 2009). Further analysis of the data using Cox Proportional Hazard (CPH), Weibull parametric and Bayesian parametric regression however showed that widowers were more likely to be disease-free, with the Bayesian result presenting greater precision as shown in its narrower credible interval. The highest median survival time observed in this study was among the CaP patients from the north central region, this could be attributable to distance patients had to travel to receive treatment, making them better prepared and financially prepared to cover all medical bills and as such receive better care and longer survival. This opinion was also shared by (Zhang \& Lawson, 2012) Most patients either drinks alcohol or smokes tobacco, these are known determinants for lung cancer, but it is not yet known if it could be defining in CaP survivorship (Peisch et al., 2017).

Diagnosis via DRE was popular among attending physician, closely followed by biopsy, this is because a DRE can indicate whether a prostate biopsy is recommended for a patient, especially in more aggressive cases (James, 2014). DRE diagnosis increased survivorship time, this could be due to its ability to quickly detect hardness of the prostate indicating the presence of CaP. DRE is known to be an independent marker for better prognosis (Migowski, 2010), however this was not consistent with this study. It was observed under the Bayesian analysis that diagnosis via transrectal ultrasound, biopsy and a combination of DRE and biopsy were markers for better survival prognosis. 
The absence of a parent, or first-degree male relative with a known case of $\mathrm{CaP}$ did impact the likelihood of experiencing remission, given the genetics and hereditary factors that come to play from one generation to another it further highlight that they is every likelihood of this disease not recurring in another generation and in agreement with an earlier finding (P A Kupelian, V A Kupelian, J S Witte, R Macklis, 1997).

A well differentiated Gleason group was associated with better prognosis however moderately and poorly differentiated Gleason group was associated with poor disease prognosis as found in the all three analysis, with the Bayesian analysis appearing more precise as can be shown in its narrower credible interval. It was also observed that a large proportion of patients presented with either a moderately or poorly differentiated Gleason score, this could be due to late presentation and lack of adequate awareness on the importance of frequent medical check-ups and early presentation (Afriansyah, Rizal, Hamid, Mochtar, \& Umbas, 2018; Khan, 2011). As also reported in studies by (Titilola O Akinremi, Ogo, \& Olutunde, 2011) most patients presented at an advanced stage which is a recurring theme among Nigerian patients. This may be a case of negligence and lack of regular awareness on the part of policy-makers on the part of patient's, distance and cost of receiving care may be responsible. As shown in this study late presentation was also a contributing factor for poor survival prognosis and patients in this category often experience recurrence (Migowski, 2010) this is consistent with findings in Ghana (Diaz, Lally, \& Pollack, 2010). This shows that early presentation particular at stage I, could increase a patient's chance of being disease-free.

Most patients presented with metastasis especially to the spine and others with multiple metastasis (Titilola O Akinremi et al., 2011; Jedy-agba, Paula, Ogunbiyi, Oga, \& Fabowale, 2012). It has been observed that before diagnosis, patients usually go through a series of assumptive diagnosis such that before a disease is detected it has metastasized beyond the prostate region. Much more effort is needed particularly by policy makers to ensure quick diagnosis and frequent screening practices to detect this disease early. Paramount to survivorship is the management of CaP as most patients still experience residual and possible recurrence given the spread of this disease to advance areas, especially in the case of multiple metastasis. 
An elevated PSA level at diagnosis is usually associated with poor disease prognosis, although due to differences in technical performance and reference standards, PSA measurements from different labs are not necessarily comparable, however approximately $98 \%$ of patients with metastatic $\mathrm{CaP}$ will have elevated PSA (Yang \& Armstrong, 2010). In this study though, PSA level did not affect treatment outcome across the $\mathrm{CPH}$, parametric and Bayesian models. Observed among patients in this facility was the inconsistency of PSA levels during treatment. This could be due to lack of proper care and lack of finance for consistent treatment on the part of patients, as some patients may miss a certain number of treatments before resuming treatment.

As observed in the CPH model patients who received chemotherapy and surgery were less likely to experience remission, this was also observed in the Weibull and Bayesian parametric regression. Patients who received radiotherapy alone as observed in the Bayesian analysis experienced being disease-free earlier. However only a few patients received this treatment regimen, this could be due to unavailability of a radiotherapy machine, coupled with frequent breakdown and workers industrial action (Gillessen et al., 2018; Robinson et al., 2018; Wolff et al., 2015).

In comparing the $\mathrm{CPH}$, parametric Weibull and Bayesian Parametric models, the Bayesian approach performed better than other models by extracting the most significant predictors particular in the 2010 - 2014 cohort. Some of significant factors were in agreement with those found in (Balogun, Role, \& Dawodu, 2014; Migowski, 2010); it was observed that the Bayesian approach is a more appealing alternative to analyzing and reporting time-to-event studies (Zhang \& Lawson, 2012) as compared to traditional frequentist approach, due to providing a flexible framework for smoothing rates and representing statistical uncertainty in rate ratios (Chen et al., 2013). Although still used sparingly, the Bayesian approach is fast gaining recognition due to advancement in computing power and software as found in this study.

\section{Limitation of the study}

In an effort to carry out this research, the researcher was faced with the challenge of extracting the required data for the study as it involves review of patient medical records; of great concern was the limitation of proper record keeping and lack of follow-up as the true state of most patient could 
not be determined. An important limitation is lack of records on patient's com-morbid status, lifestyle and behavioural variables.

\section{Reference}

Abdulkareem, F. (2009). Epidemiology \& Incidence of Common Cancers in Nigeria. Cancer Registry and Epidemiology Worshop April 2009, 1-58.

ACS. (2016). What Is Prostate Cancer? Facts \& Figures.

Afriansyah, A., Rizal, A., Hamid, A. H., Mochtar, C. A., \& Umbas, R. (2018). Survival analysis and development of a prognostic nomogram for bone-metastatic prostate cancer patients : A single-center experience in Indonesia. International Journal of Urology, 1-7. https://doi.org/10.1111/iju.13813

Akinremi, T. O., Adeniyi, A., Olutunde, A., Oduniyi, A., \& Ogo, C. N. (2014). Need for and relevance of prostate cancer screening in Nigeria. Ecancermedicalscience, 6-11. https://doi.org/10.3332/ecancer.2014.457

Akinremi, T. O., Ogo, C. N., \& Olutunde, A. O. (2011). Review of prostate cancer research in Nigeria. Infectious Agents and Cancer, 6(Suppl 2), S8. https://doi.org/10.1186/1750-9378-6$\mathrm{S} 2-\mathrm{S} 8$

ASCO. (2015). Prostate Cancer. Patient Guide. Alexandria, VA. Retrieved from www.asco.org

Balogun, O. S., Role, M. R., \& Dawodu, O. O. (2014). Survival Analysis of Prostate Cancer In Ilorin, Kwara State. IOSR Journal of Dental and Medical Sciences Ver. II, 04(06), 10-19.

Bayes, T. (1763). An essay towards solving a problem in the doctrine of chances.

Berger, J. O. (1985). Statistical Decision Theory and Bayesian Analysis. Springer-Verlag.

Box, G. E. P., \& Tiao, G. C. (1992). Bayesian Inference in Statistical Analysis. Book. https://doi.org/10.1002/9781118033197

Chen, W., Armstrong, B. K., Zheng, R., Zhang, S., Yu, X., \& Clements, M. (2013). Cancer burden in China : a Bayesian approach. BMC Cancer, (Mi).

Cooney, M. M., Okuku, F., \& Orem, J. (2016). Prostate Cancer Burden at the Uganda Cancer Institute, 2(4), 181-185.

DF, G. (1992). Histologic grading of prostate cancer: A prespective. Hum Pathol, 23(273), 279.

Diaz, D. A., Lally, B. E., \& Pollack, A. (2010). A SEER Survival Analysis of High Grade Prostate Cancer: Surgery vs. Radiotherapy. Radiation Oncology Biology, 78(3), S150. https://doi.org/10.1016/j.ijrobp.2010.07.372

Fernandez, P., Zeigler-johnson, C. M., Spangler, E., Merwe, V. Der, Jalloh, M., Gueye, S. M., \& Rebbeck, T. R. (2012). Androgen Metabolism Gene Polymorphisms , Associations with Prostate Cancer Risk and Pathological Characteristics : A Comparative Analysis between South African and Senegalese Men, 2012. https://doi.org/10.1155/2012/798634

Gandenberger, G. (2014a). An Introduction to Bayesian, Likelihoodist, and Frequentist Methods Gandenberger, G. (2014b). An Introduction to Likelihoodist, Bayesian, and Frequentist Methods 
Gandenberger, G. (2014c). An Introduction to Likelihoodist, Bayesian, and Frequentist Methods Gillessen, S., Attard, G., Beer, T. M., Beltran, H., Bossi, A., Bristow, R., Omlin, A. (2018). Management of Patients with Advanced Prostate Cancer: The Report of the Advanced Prostate Cancer Consensus Conference APCCC 2017. European Urology, 73(2), 178-211. https://doi.org/10.1016/j.eururo.2017.06.002

Ibrahim, J. G., Chen, M.-H., \& Sinha, D. (2005). Bayesian Survival Analysis. Encyclopedia of Biostatistics. https://doi.org/10.1002/0470011815.b2a11006

Ifere, G. O., Abebe, F., \& Ananaba, G. A. (2012). Emergent trends in the reported incidence of prostate cancer in Nigeria. Dove Press: Clinical Epidemiology, 19-32.

Ikuerowo, S. O., Omisanjo, O. A., Bioku, M. J., Ajala, M. O., Patrick, V., Mordi, N., \& Esho, J. O. (2013). Prevalence and characteristics of prostate cancer among participants of a community- based screening in Nigeria using serum prostate specific antigen and digital rectal examination, 8688, 1-7. https://doi.org/10.11604/pamj.2013.15.129.2489

Jackson, R. J. (2015). Some Statistical Methods for the Analysis of Survival Data by.

James, N. (2014). Premier on Prostate Cancer. Springer Healthcare. https://doi.org/10.1007/9781-907673-82-5

Jedy-agba, E., Paula, M., Ogunbiyi, O., Oga, E., \& Fabowale, T. (2012). Cancer Incidence in Nigeria : A Report from Population-based Cancer Registries Cancer incidence in Nigeria : A report from population-based cancer registries. Cancer Epidemiology, (May). https://doi.org/10.1016/j.canep.2012.04.007

Ji, G., Huang, C., Song, G., Xiong, G., Fang, D., Wang, H., Zhou, L. (2017). Are the Pathological Characteristics of Prostate Cancer More Aggressive or More Indolent Depending upon the Patient Age? Hindawi, 2017.

Kgatle, M. M., Kalla, A. A., Islam, M. M., Sathekge, M., \& Moorad, R. (2016). Prostate Cancer : Epigenetic Alterations, Risk Factors, and Therapy, 2016. https://doi.org/10.1155/2016/5653862

Khan, H. (2011). DETERMINANTS OF PROSTATE CANCER: The Birmingham Prostatic Neoplasms Association Study Unit of Urologic and Genetic Epidemiology.

Migowski, A. (2010). Survival of patients with clinically localized prostate cancer. Rev Saude Publica, 44(2).

Mohammadian-hafshejani, A., Ghoncheh, M., Towhidi, F., Jamehshorani, S., \& Salehiniya, H. (2016). Incidence and mortality of prostate cancer and their relationship with the Human Development Index worldwide. Prostate International, 4(3), 118-124. https://doi.org/10.1016/j.prnil.2016.07.001

Oakes, D. R. C. and D. (1972). Analysis of Survival Data. Chapman \& Hall/CRC.

Odedina, F. T., Akinremi, T. O., Chinegwundoh, F., Roberts, R., Yu, D., Reams, R. R., Kumar, N. (2009). Prostate cancer disparities in Black men of African descent : a comparative literature review of prostate cancer burden among Black men in the United States, Caribbean, United Kingdom , and West Africa. Biomed Central, 8, 1-8. https://doi.org/10.1186/1750-9378-4S1-S2 
P A Kupelian, V A Kupelian, J S Witte, R Macklis, and E. A. K. (1997). Family history of prostate cancer in patients with localized prostate cancer: an independent predictor of treatment outcome. Journal of Clinical Oncology, 15(4).

Peisch, S. F., Blarigan, E. L. Van, Chan, J. M., Stampfer, M. J., Kenfield, A., Medical, H., \& Francisco, S. (2017). Prostate cancer progression and mortality: a review of diet and lifestyle factors Sam. World J Urol, 35(6), 867-874. https://doi.org/10.1007/s00345-016-19143.Prostate

Robinson, D., Garmo, H., Lissbrant, I. F., Widmark, A., Pettersson, A., Gunnlaugsson, A., ... Stattin, P. (2018). Prostate Cancer Death After Radiotherapy or Radical Prostatectomy: A Nationwide Population-based Observational Study. European Urology, 73(4), 502-511. https://doi.org/10.1016/j.eururo.2017.11.039

Sunny, L. (2005). An Epidemiological Study in India.

Wolff, R. F., Ryder, S., Bossi, A., Briganti, A., Crook, J., Henry, A., ... Kleijnen, J. (2015). A systematic review of randomised controlled trials of radiotherapy for localised prostate cancer. European Journal of Cancer, 51(16), 2345-2367. https://doi.org/10.1016/j.ejca.2015.07.019

Yang, S. C., \& Armstrong, D. K. (2010). Early Diagnosis and Treatment of Cancer.

Zhang, J., \& Lawson, A. B. (2012). Bayesian Parametric Accelerated Failure Time Spatial Model and its Application to Prostate Cancer. J Appl Stat, 38(2), 591-603. https://doi.org/10.1080/02664760903521476.Bayesian 